\title{
Ultrasoft microwire neural electrodes improve chronic tissue integration
}

Zhanhong Jeff Du, ${ }^{\text {a,b,c,f*, }}$ Christi L. Kolarcik ${ }^{\mathrm{a}, \mathrm{b}, \mathrm{c}, \mathrm{d}^{*}}$, Takashi D.Y. Kozai ${ }^{\mathrm{a}, \mathrm{b}, \mathrm{c}, \mathrm{e}}$, Silvia D. Luebben ${ }^{\mathrm{g}}$, Shawn A. Sapp ${ }^{\mathrm{h}}$, Xin Sally Zheng ${ }^{\mathrm{a}}$, James A. Nabity ${ }^{\mathrm{i}}$, and X. Tracy Cui ${ }^{\mathrm{a}, \mathrm{b}, \mathrm{c}}$

${ }^{\mathrm{a}}$ Department of Bioengineering, ${ }^{\mathrm{b}}$ Center for the Neural Basis of Cognition, ${ }^{\mathrm{c}}$ McGowan Institute for Regenerative Medicine, ${ }^{\mathrm{d}}$ Systems Neuroscience Institute, ${ }^{\mathrm{e}}$ NeuroTech Center of Brain

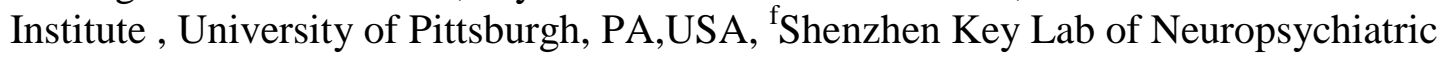
Modulation, CAS Center for Excellence in Brain Science, Shenzhen Institutes of Advanced Technology, Chinese Academy of Sciences, Shenzhen, China. ${ }^{\mathrm{g}}$ Boulder Scientific Company, Longmont, CO,USA, ${ }^{\mathrm{h}}$ Aei Corporation, Littleton, CO,USA, ${ }^{\mathrm{i}}$ Department of Aerospace Engineering Sciences, University of Colorado, Boulder, CO,USA.

Corresponding author: X. Tracy Cui, Ph.D., Department of Bioengineering, University of Pittsburgh, 5057 Biomedical Science Tower 3, 3501 Fifth Avenue, Pittsburgh, PA 15260, Ph: 412-383-6672, FAX: 412-648-9076, Email: xic11@ pitt.edu

* These authors contributed equally to this work.

Key Words: conducting elastomer, composite bio-electrodes, deep brain stimulation, neural electrode 


\begin{abstract}
Chronically implanted neural multi-electrode arrays (MEA) are an essential technology for recording electrical signals from neurons and/or modulating neural activity through stimulation. However, current MEAs, regardless of the type, elicit an inflammatory response that ultimately leads to device failure. Traditionally, rigid materials like tungsten and silicon have been employed to interface with the relatively soft neural tissue. The large stiffness mismatch is thought to exacerbate the inflammatory response. In order to minimize the disparity between the device and the brain, we fabricated novel ultrasoft electrodes consisting of elastomers and conducting polymers that had mechanical properties much more similar to those of brain tissue than previous neural implants. In this study, these ultrasoft microelectrodes were inserted and released using a stainless steel shuttle with polyethyleneglycol (PEG) glue. The implanted microwires showed functionality in acute neural stimulation. When implanted for 1 or 8 weeks, the novel soft implants demonstrated significantly reduced inflammatory tissue response at week-8 compared to tungsten wires of similar dimension and surface chemistry. Furthermore, a higher degree of cell body distortion was found next to the tungsten implants compared to the polymer implants. Our results support the use of these novel ultrasoft electrodes for long term neural implants.
\end{abstract}




\section{Introduction}

Implantable neural electrodes or multielectrode arrays (MEAs) can record neuronal activity from individual or small populations of neurons [1]. Neural signals recorded on implantable MEAs provide valuable information for systems neuroscience research, enabling researchers to gain insight into functional neural networks used in sensory processing [2], motor control [3] and cognitive functions [4]. Neural signals recorded can also be utilized clinically to control brain-computer interfaces (BCI) for the restoration of lost neural functions [5-12] or to understand the basis of neurological dysfunction [13, 14]. Alternatively, electrical signals can be delivered by MEAs to modulate neural network activity, in order to substitute lost sensory input [15] or to treat the symptoms of a neural disease. In recent years, such neural stimulation treatments have expanded to include not only motor dysfunction in Parkinson's disease [16] and neural network hyperactivity in epilepsy [17] but mental disorders such as depression [18] and obsessive compulsive disorder (OCD) [19].

One of the major challenges limiting the widespread use of neural implants in clinical applications is that these chronic devices fail over time. In the monkey cortex, previous reports indicated a $40 \%$ drop in the number of functional electrodes within 30 days [20]. The recorded action potential amplitude was also reported to decrease at the rate of 2.4\% per month [21], which detrimentally impacts the outputs of decoding algorithms used in BCI [1]. Similar device performance degradation is observed in rodent studies [22-27]. Likewise, current clinical deep brain stimulation (DBS) implants could benefit tremendously from improved electrode technology. The DBS electrode surface impedance increases rapidly following implantation due to scar tissue encapsulation [28-30]. This decreases the effective range of the DBS electrode, increases battery drainage and causes variation in therapeutic effects [31]. Conventional DBS electrodes display large trial-to-trial variation which cannot be explained by known mechanisms [32]. Despite the decrease of impedance following neural stimulation [30, 33], possibly caused by the disruption of tissue encapsulation, the heightened and varying electrode impedance could be the cause of these unpredictable and variable neural stimulation effects.

One proposed primary mechanism of such device failure is the foreign body response of the brain $[34,35]$. MEA implantation damages the blood brain barrier (BBB) and exposes a portion of the brain to substances that are typically outside this barrier [36, 37]. This triggers an acute immune response from both microglia and astrocytes [38-40], which is then followed by chronic inflammation and neural degeneration around the implant [35, 41, 42]. These reactions can cause neuronal death and neurite degeneration and result in the formation of a tight glial sheath that blocks the electrical current flow for recording and stimulation [43]. Persistent leakage of the BBB can occur and may contribute to the accumulation of neurotoxic factors that negatively impact neuronal health and device performance [44]. It is also important to note that a significant amount of implantation variability is observed [26, 37, 45, 46]. As a result, the complex causal mechanisms behind device failure remain poorly understood.

The magnitude of the tissue response following device insertion could be influenced by a

wide range of physical characteristics $[35,47]$ including the surface and bulk properties of the 
device. Surface factors include hydrophilicity $[9-11,48]$, texture $[49,50]$ and presence or absence of bioactive molecules [51-53]. Bulk properties include size and geometry of the device [47, 48, 54-56] as well as the stiffness of the implanted materials [57]. Current neural probes are fabricated with very rigid materials with a high Young's modulus (E) such as tungsten ( $E=400$ $\mathrm{GPa})$, silicon $(\mathrm{E}=200 \mathrm{GPa})$, polyimide $(\mathrm{E}=3 \mathrm{GPa})$ or parylene $\mathrm{C}(\mathrm{E}=2-5 \mathrm{GPa})$, while the brain tissue is very soft $(\mathrm{E}=0.4-15 \mathrm{kPa})$ [58-61]. The enormous elastic modulus mismatch could contribute to "frustrated phagocytosis" [62-64] and exacerbate friction-induced stress under micromotion [38, 57, 65-67]. Furthermore, pro-inflammatory interleukin-1 $\beta$ (IL-1 $\beta$ ) is shown to be upregulated by increased mechanical strain [68], which impairs the chronic performance of in vivo MEAs [34, 64]. The glial scar, neuronal health and overall cellular response at the electrodetissue interface are all negatively impacted by the mechanical mismatch between the implant and the brain tissue [34, 43, 63, 69, 70]. Moreover, stiff implants with delicate designs cannot adapt to normal tissue deformations as occurs with any implant, another factor that may lead to device failure from material fatigue and degradation [23].

Finite element modeling predicts that softer materials for neural implants can reduce the mechanical damage at the device-tissue interface and the micromotion-induced strain $[57,66]$. For this reason, compliant materials [71, 72] and mechanically-adaptive materials [73, 74] have been evaluated in both MEA shanks [45, 75] and electrode coating materials [76]. Despite efforts to increase the softness and flexibility of in vivo MEAs, the Young's modulus of previously studied implants was only reduced to $5 \mathrm{MPa}$ [77] 12MPa[78] or $15 \mathrm{MPa}$ [79]. To develop a softer implant that more closely mimics the mechanical properties of brain tissue, we fabricated ultra-soft microwires. The electrically conducting core of the microwire is made with a poly(3,4ethylenedioxythiophene)-poly(ethyleneglycol) copolymer [PEDOT-PEG] with poly(dimethylsiloxane)[PDMS] elastomer; the electrically insulated outer coating is made of fluorosilicone [80]. The complete microwire electrode had a Young's modulus of $974 \mathrm{kPa}$ and the ratio of PEDOT/PDMS was optimized for sufficient conductivity while maintaining the flexibility of the material [80].

Due to the difficulty of precisely implanting flexible devices into a target tissue location with currently available techniques, most recent flexible electrode arrays have been studied only in vitro $[71,81]$ or via surface electrical recording and stimulation $[75,82,83]$. Several methods have been developed to deliver soft and flexible microdevices into neural tissue [71, 84], or the mechanical stiffness of implant could be adaptive depending on the condition of implants [78]. In this study, we used a stiff shuttle to support and guide the microwire to the target location. The shuttle was made of a stiff needle that detached from the implant in vivo after precise targeting to the designated brain region.

Here, we report on the in vivo biocompatibility of our ultrasoft wire implants in the rodent brain for 1 and 8 weeks with comparisons, to tungsten microwires, commonly used in neural electrode arrays. To accurately assess the mechanical effect of these soft wires on the brain tissue response over control implants, both types of implants were designed to have similar geometry and surface chemistry. In addition to the common quantitative immunohistochemical 
analysis on inflammation, glial scaring, BBB injury, and neuronal health and density [85], a novel automated cell shape and strain analysis was developed, which demonstrates the striking difference in neuronal cell shape and tissue strain around the soft and stiff implants. Lastly, neural stimulation was performed in rat subthalamic nucleus (STN) to demonstrate the functionality of the soft electrodes for DBS applications [86, 87].

\section{Methods}

\section{Fabrication of electrodes}

The implantable conducting elastomer microwire was reported in our previous study [80]. The fabrication process is briefly described as follows:

1) The soft conducting wires were made by extruding a blend of PEDOT-PEG conducting polymer (Aedotron ${ }^{\mathrm{TM}} \mathrm{C} 3$, TDA Research Inc.) and polydimethylsiloxane (MED6607, NuSil Technology) through a $29 \mathrm{G}$ syringe needle. The wires were sputter-coated with gold along the shaft to increase the conductivity. Then, the wires were dip-coated up to 5 times to build an approximately 5- $\mu \mathrm{m}$-thick layer of fluorosilicone (MED6655, NuSil Technology) along the outer surface that insulated the 125 - $\mu$ m-diameter soft wire. The wire was trimmed at both ends with a razor to expose the conductive tips with active area of $12270 \mu \mathrm{m}^{2}$ (one exposed end shown in inset of Fig. 1).

2) Standard tip tungsten microelectrodes with $127 \mu \mathrm{m}$ diameter (MicroProbes, Gaithersburg, MD) were used as controls. To match the surface chemistry, the tungsten wires were dip-coated with a $380 \mathrm{~nm}$ thick fluorosilicone layer (resulting in a final outer diameter of $127.76 \mu \mathrm{m}$ ). After coating, tungsten electrodes were cut with wire cutters to match the squared-off tip profile of the soft wires.

The soft polymer wire had a Young's modulus of E=974 $\mathrm{kPa}$, cross section area A of $12270 \mu \mathrm{m}^{2}$, and a length $\mathrm{L}$ of $1.5 \mathrm{~cm}$, thus the axial stiffness $\mathrm{k}$ of the wire defined by equation (1) was $0.797 \mathrm{~N} / \mathrm{m}$. With a Young's modulus of $\mathrm{E}=400 \mathrm{GPa}$, a cross section and active area of $12820 \mu^{2}$ and a length of $1.2 \mathrm{~cm}$, the Tungsten wire has a stiffness of $0.427 \times 10^{6} \mathrm{~N} / \mathrm{m}$, which is six orders of magnitude higher than the ultrasoft polymer wire. The geometrically soft polymer wires and geometrically stiff or rigid tungsten wires are therefore referred to as soft and stiff wires throughout the remainder of this manuscript, respectively.

$$
k=\frac{A * E}{L}
$$

\section{Fabrication of shuttles for implantation}

Figure 1 illustrates the shuttle fabrication steps and features to support implantation of wire electrodes. 3.8-cm long 27G hypodermic dental needles (Fen Dental, Hialeah, FL) were used to make the insertion shuttles. The LuerLok hub was removed and the needle glued to a polyester polishing block using a UV-curable adhesive (\#68 Norland Optical Adhesive, Edmund Optics). Block-mounted needles were hand-polished on a Buehler polishing wheel with a $20 \mu \mathrm{m}$ diamond lap surface. This slowly removed the top portion of the hypodermic needle until the 
center hollow was exposed and subsequently thinned down to just past the axial mid-point. After physically releasing the needles from the adhesive, the semi-cylindrical opening was used to cradle the soft wires during insertion.

Microwires (both soft and stiff) and shuttles were treated with oxygen plasma [71] for 120 seconds immediately prior to implantation to increase the hydrophilicity of the surface. Wires were then glued into the shuttle cavity with a $45 \%$ wt. solution of PEG (average molecular weight $=1500$ Dalton) in acetonitrile to build the implant assembly (Fig. 1). The PEG glue was designed to hold the soft wire and shuttle together during insertion but to quickly dissolve within about 30 seconds after implantation to enable removal of the shuttle while leaving the microelectrode in place (although tweezers were used to stabilize the soft wire microelectrode near the brain surface preventing it from being pulled out during extraction of the shuttle). To keep the trauma to the insertion site nearly the same, stiff wires were also glued to the shuttles. Tweezers were not needed to hold the stiff wire electrodes in place while removing the shuttle.

\section{Surgical procedures for biocompatibility studies}

10 weeks male Sprague-Dawley rats (Charles River) weighing 300-350g were utilized to evaluate the in vivo performance of soft wires compare to conventional stiff wires. Animals were anesthetized with $3.0 \%$ isofluorane in $0.8 \mathrm{~L} / \mathrm{min}$ oxygen for 5 minutes prior to surgery and then maintained for the duration of the procedure at $2.25 \%$ isofluorane. Anesthesia level was monitored closely during the procedure by observing changes in respiratory rate, heart rate, body temperature $\left(37.7^{\circ} \mathrm{C}\right.$ ) and absence of the pedal reflex. Animals were placed in a stereotaxic frame and the hair removed over the incision site. In each animal, four wires were implanted, two soft wires were randomly implanted into left or right hemisphere and two stiff wires were implanted into the contralateral hemisphere. Wires were at least $2 \mathrm{~mm}$ apart on the skull to prevent possible crosstalk between tissue reactions around each wire. Wires were implanted to target a depth of $8 \mathrm{~mm}$ from the cortical surface with the aid of a stainless steel half needle insertion shuttle glued to the wire with PEG as described above. Once implanted, the PEG dissolved allowing the shuttle to detach from the wire and be removed. This design was used to insert both soft and stiff wires. Blue light-curing dental cement was used to build a well on the craniotomies, and craniotomies were filled with Kwik-Sil with the wires protruding out of the dental cement wells. Protruding ends of the wires were tethered to the skull with dental cement. Screws were placed to anchor the headcap. Acute (week-1, $n=7$ ) and chronic (week-8, $n=7$ ) time points were evaluated, number of analyzed images per group were varied due to either outlier removal or analyzing multiple depth per animal, thus the image $n$ per group are listed in each figure. All surgical procedures were done in accordance with the guidelines outlined by the United States Department of Agriculture and approved by the Institutional Animal Care and Use Committee of the University of Pittsburgh. Animals were housed in the facilities of the University of Pittsburgh, Department of Laboratory Animal Resources and given free access to food and water. 
DBS with soft wires evoke Electroencephalography (EEG) Local Field Potential(LFP)

Animals were anesthetized with $3.0 \%$ isofluorane in $0.8 \mathrm{~L} / \mathrm{min}$ oxygen and maintained at $2.25 \%$ isofluorane. Soft wires $(n=2)$ were implanted into the STN of rats $(3.8 \mathrm{~mm}$ posterior to bregma, $2.5 \mathrm{~mm}$ lateral to midline, $8 \mathrm{~mm}$ deep from brain surface) through a craniotomy to perform DBS and evoke reliable EEG LFP signal as outlined in previous studies [86]. The dura mater was cut to prevent possible separation of the soft wire from the shuttle due to the high friction at the dura surface. The soft wire was delivered using the shuttle in order to evoke specific neural activity in the STN neural network. An EEG recording screw was placed on the motor cortex $(+2.5 \mathrm{~mm}$ anterior to bregma, $+2.5 \mathrm{~mm}$ lateral to midline) and the EEG ground screw was positioned above the cerebellum $(0.8 \mathrm{~mm}$ posterior to lambda and $2.0 \mathrm{~mm}$ lateral to midline). First, an impedance spectrum of the soft wire was measured after the electrode was implanted in the STN using the stainless steel half needle as counter electrode (Fig. 7A). The stainless steel half needle shuttle was retained in the tissue as the counter electrode to perform bipolar stimulation. Cathodic leading bi-polar square-wave stimulation with $90 \mu \mathrm{A}$ amplitude and $200 \mu$ s duration per phase was delivered at $130 \mathrm{~Hz}$ to evoke LFP in motor cortex. A total of 650 waveforms were averaged to obtain the mean evoked LFP from the stimulation. After one DBS experiment, the rat was immediately euthanized with intracardial potassium chloride injection and further stimulations were performed to validate that the evoked LFP response earlier was not an artifact.

\section{Histology}

At the designated time points, animals were deeply anesthetized with a ketamine/xylazine cocktail $(100 / 20 \mathrm{mg} / \mathrm{kg}$ ) via the intraperitoneal (IP) cavity. Animals were then transcardially perfused with cold $\left(4^{\circ} \mathrm{C}\right)$ PBS followed by $4 \%(\mathrm{w} / \mathrm{v})$ paraformaldehyde (PFA) in PBS. For soft wires, rongeurs were used to carefully remove the bone and dental cement and expose the craniotomy. Then the soft wires were cut with scissors above the brain surface. All stiff wires were extracted together with the headcaps. Majority of the soft wires were left in the brain during tissue processing and sectioning with the exception of 2 soft wires being extracted for imaging of wire surface. The brain was removed, post-fixed for up to 3 days and then equilibrated in $30 \%$ sucrose (used as a cryoprotectant) prior to freezing. Serial sections were cut at a $15 \mu \mathrm{m}$ thickness. The preserved soft and stiff wires were glued on metal studs and sputtered with $3.5 \mathrm{~nm}$ Pd using a Cressington Sputter Coater. Wire surface was imaged under JSM 6330F SEM (Joel, Japan) for characterization of cell adhesion onto the electrode.

For immunofluorescence staining, tissue sections were stained at the same time for each antibody combination to minimize variability. Markers to visualize mature axons (1:500 rabbit anti-NF-200, Abcam, Cambridge, UK), microglia (1:500 rabbit anti-Iba1, Wako Chemicals USA, Richmond, VA), astrocytes/fibroblasts/endothelial cells (1:500 anti-Vimentin, Clone V-9, Millipore, Billerica MA), astrocytes (1:500 rabbit anti-GFAP, Dako, Glostrup, Denmark), neuronal nuclei (1:500 mouse anti-NeuN, Millipore, Billerica MA), apoptotic cell death (cleaved caspase-3, 1:50 Asp175; Cell Signaling Technology, Boston, MA), L1(a kind gift from Dr. Carl Lagenaur) and blood-brain barrier injury (1:500 goat anti-rabbit IgG Alexa 633 conjugated, Invitrogen, Carlsbad CA), (antibodies outlined in Table 1) were used. 
Tissue sections were hydrated in PBS and non-specific binding blocked with $0.5 \%$ BSA. Primary antibodies were then diluted in BSA and added for approximately 1 hour. After washing with BSA, fluorophore-conjugated secondary antibodies (conjugated to Alexa Fluor 488, Alexa Fluor 568 or Alexa Fluor 633) diluted in BSA were added for approximately 1 hour and Hoechst used as the nuclear stain. Fluoromount-G (Southern Biotechnology Associates, Birmingham, AL) was used for mounting and to preserve fluorescence. Negative controls lacking primary antibody or including the appropriate blocking peptide were included for each secondary antibody or primary antibody, respectively.

\section{Quantitative tissue analysis}

Confocal fluorescent microscopy was used to evaluate the cellular reactions associated with the implanted electrodes. Images were acquired using an Olympus Fluoview 1000 II Confocal Microscope (Olympus America, Center Valley, PA) at the Center for Biologic Imaging at the University of Pittsburgh. For each antibody, images were acquired using the same laser power, exposure time, and detector settings to reduce variability during data analysis. Images were centered on the implant site and multi-channel images were acquired simultaneously. Fluorescent images of the explanted wires were reconstructed from 11 z-stack images with a step size of $4.1 \mu \mathrm{m}$ in order to compensate for the curved surface of the wire. Pixel-based image intensity analytics were performed using previously published custom MATLAB script I.N.T.E.N.S.I.T.Y. v2.0 [85] .

Briefly, to prevent holes in the tissue (major blood vessels and/or shuttle tracks) from artificially reducing the average activity-dependent fluorescence, background noise intensity thresholds were calculated from corresponding control images. To calculate the background noise intensity threshold, pixels with intensity greater than one standard deviation dimmer than mean pixel intensity were removed from the calculation. The threshold was then determined by calculating the pixel intensity of one standard deviation below the mean of the remaining pixel intensities. Bins with intensity values dimmer than average intensities of the control images were considered tissue "holes." Using MATLAB, the center of the shuttle track was identified on each image, after which the script generated masks of concentric rings. The average intensity for all pixels above the background noise intensity threshold in each bin was calculated and normalized against the background to calculate the Signal-to-Noise Intensity Ratio (SNIR) in each bin as outlined in equation (2);

$$
S N I R=\frac{A v g I_{>T}}{A v g N}
$$

where $A v g I_{>_{T}}$ is the mean Intensity of all pixels above the noise threshold $(>T)$ in each bin, and $\operatorname{AvgN}$ is the mean noise floor intensity. This means an SNIR of 1 represents the noise floor. Therefore, it is expected that the SNIR does not asymptote to 1 unless there is no staining signal in the corresponding bin. Data were averaged for each implant type and time point, and then reported as mean \pm standard error. 


\section{Quantification of mechanical strain-induced cell deformation}

To quantitatively assess the cell body shape deformation caused by the presence of a nearby foreign body (i.e., microelectrode), NeuN images were pre-processed and the cell shape was extracted using custom MATLAB scripts. To enhance cell shape recognition and reduce artifact-related false positives, images were flattened using a custom MATLAB script. First, image noise thresholds were established as with the I.N.T.E.N.S.I.T.Y. Analyzer v2.0 to create a mask to remove holes in the tissue. Next, a two-dimensional discrete Fourier Transform was applied over the remaining region and the zero-frequency component shifted to the center of the image. Once the image was flattened, the longer and shorter axes of the cell were determined by the program; the angle between the longer axis of the cell and the radial line from implant center to cell center was utilized to determine the direction of cell shape deformation. This angle was termed the cell elongation angle (CEA) and indicated the direction in which the cell body undergoes the most strain. That is, an angle near $90^{\circ}$ indicates that the cell is most affected by mechanical strain from the radial direction outward from the implant. Conversely, a CEA near $0^{\circ}$ indicates that the strain occurs predominantly from the tangential direction of the center-out line. The extent of cell body shape deformation was defined as the cell shape strain index (CSSI) as described in equation (3):

$$
C S S I=\frac{\Delta L}{L_{0}}=\frac{(b-a)}{\frac{(b+a)}{2}}
$$

Where $b$ is the length of the longer axis of the cell as defined in an elliptical shape, and a is the length of the shorter axis. $\Delta L$ represents the mechanical strain that a neuronal nuclei experiences, while $L_{0}$ estimates the mean diameter of the neuronal nuclei in the absence of strain. The mechanical strain associated with probe volume as well as the relationship between distance from the implant and CEA were determined.

\section{Statistics}

In all analysis the error bars represented the standard error of the sample. For cell number analysis, two-sample T-test was performed to compare the differences between soft and stiff wires. For the cell shape deformation analysis with CEA and CSSI comparison, Wilcoxon rank sum test was performed to compare the two groups. For all tests, a $<0.05$ indicated a statistically significant difference.

\section{Results}

Sub-chronic and chronic inflammatory tissue response.

In order to evaluate the inflammatory tissue response and axonal damage associated with the implanted microelectrodes, the amount and distribution of microglia (Iba-1), astrocytes (GFAP) and axons (NF-200) were visualized. The soft wire tissue interface was well-preserved

(Fig. 2) as soft wires were not explanted prior to sectioning of the fixed brain. On the other hand, even though the stiff wires have circular cross section and the diameter closely mimics the soft wire, the holes in the tissue sections are often irregular in shape (Fig. 2). Since stiff wires had to 
be extracted before tissue processing and fixation, tissue friction during explantation and the slight stretching of brain slice during mounting could cause the distortion. The fixation process and the sucrose dehydration process could also each contribute to the distortion to some extent. At the week-1 time point, less glial activation was observed around soft wires as compared to stiff wires (Fig. 2, Compare E vs. F and I vs. J) although no statistical significance was noted (Fig. 2, S and U). The NF200 image demonstrated the same feature (Fig. 2, Compare A vs B) but yielded statistically significant differences in two quantified bins (Fig. 2, Q). This difference diminished at week-8 for NF200 intensity (Fig. 2, R). The inflammatory response around both types of implants was distributed over a relatively large region around each implant to a similar degree, indicating that the soft wire implants do not significantly reduce the acute tissue response. This is to be expected as both experimental and control tissues were damaged by the shuttle and at 7 days the stab wound may not be fully recovered. However, at week-8, a binned Iba-1 staining intensity reduction as much as $18.9 \%$ was observed around soft wires compared to the stiff ones (Fig. 2, G vs. H) and the area associated with the glial sheath was decreased when compared to that around the stiff implants (Fig. 2, K vs. L). Consider GFAP relative intensity of 2 as the threshold for defined inflammation, at week-8, soft wire had 17 bins over threshold while stiff wires had 26 bins over threshold, yielding a strikingly $49.9 \%$ reduction in glial sheath area. These differences were statistically significant at distances within $100 \mu \mathrm{m}$ from the interface (Fig. 2, $\mathrm{T}$ and V) and a much greater difference was observed in GFAP intensity. In addition, the intensity of GFAP and Iba-1 staining immediately around the soft wire implants is similar to the intensity observed in healthy tissue (i.e., at locations far from the implant site) (Fig. 2, $\mathrm{G}$ and $\mathrm{K}$ ), which suggests an interface that does not continue to elicit an inflammatory response at this longer time point. In comparison, significant glial scarring consisting of both microglia and astrocytes formed around stiff implants as previously reported[41, 52] (Fig. 2, H and L).

\section{Neuronal density and health around the implant}

To characterize the extent of neuronal loss near the implant, NeuN staining was combined with staining for activated caspase- 3 to identify neurons undergoing programmed cell death via apoptosis [6]. Only a small number of apoptotic neurons indicated by colocalization of NeuN and caspase-3 were observed in all groups examined (Table 1), while a much larger number of non-neuronal cells were positive for caspase-3 (Fig. 3 A-D). Neuron numbers were quantified with automated MATLAB script. At week-1, the cell density around the soft implants was the same as for the stiff implants (Fig. 3, M). At week-8, however, the number of neurons around soft implants was significantly higher than those around the stiff implants at distances of 60 90 and 120 210 $\mu \mathrm{m}$. The neuron density around the soft implants between 90-120 $\mu \mathrm{m}$ still demonstrated a clear trend of increase compared to the stiff implants, although not statistically significant. The density of neurons can be highly variable according to many factors, including the heterogeneous neuron density in the brain region before implantation, the kill zone of host 
tissue response, the slice thickness, the mechanical distortion of implant-tissue interface as well as the bin size for post hoc analysis. Higher sample size may likely result in statistical significance in this $30 \mu \mathrm{m}$ zone. Nevertheless, the NeuN positive cell numbers in all other quantified bins were higher around the soft implant than the stiff implant. This phenomenon combined with the sparse caspase- 3 positive cells suggests that the soft implants maintain a favorable environment for the survival of neurons compared to the stiff implants.

\section{Cell deformation reflects different device-dependent strain}

To better characterize the effects of implant softness on neuronal cells, cellular morphology was further characterized using both CEA and CSSI calculations. Three control images (from non implanted brain regions of three animals at the similar depth as the experimental groups) were analyzed. The mean CEA of control tissue was calculated to be 44.2 \pm 0.6 . For cells in an unaltered mechanical environment, CEA values ranging from $0-90^{\circ}$ with a mean of $45^{\circ}$ would be expected. The CSSI was determined to be $0.383 \pm 0.006$, reflecting the fact that the shape of the sectioned neuronal bodies are mostly non-circular. The CEA and CSSI from the control images gave the benchmark for non mechanically strained cell shape. Then experimental images were analyzed and quantified cells were binned every $30 \mu \mathrm{m}$ beginning $30 \mu \mathrm{m}$ from the tissue-electrode interface. The bins were determined such that the first bin had enough cells for quantification and statistical evaluation. At week 1, the CEA of cells closer to the soft wire implants showed higher CEA than non implanted control and this difference disappears beyond $120 \mu \mathrm{m}$. (Fig. 4C). On the other hand, the cells around both soft and stiff implant showed significantly greater CSSI than the non implanted control of $0.383 \pm 0.006$ in most bins (Fig. 4D). Cells in the first quantifiable bin showed higher CEA in the presence of soft wire implants (Fig. 4C-D), possibly due to mechanical perturbations by the shuttle during soft wire implantation. Because soft wires were retained in the brain tissue by friction when shuttles were removed, tweezers were often used to stabilize the wire near the brain surface to retrieve the shuttle. During this process, additional strain was inevitably applied to cells surrounding the implant. This differs from what was done with the stiff wires as these wires could be held with greater stability during shuttle extraction. Interestingly, this effect was reversed at week-8 time point (Fig. 4E-F) when both the CEA and CSSI are as much as 20.2\% and $31.6 \%$ higher in the presence of stiff wires than in the presence of soft wires, respectively. This suggests that cells near soft wires eventually recover from initial implant strain while cells near stiff wire are persistently deformed over the course of these experiments. The CSSI difference between the two groups were roughly consistent at week-1 and week-8, however, the difference was much great at week-8 with statistical p-value as small as $4.79 * 10^{-13}$.

\section{$B B B$ injury related inflammation}

We also examined the distribution of vimentin-positive cells (fibroblasts and glia cells), neural adhesion molecule L1 and the extent of blood-brain barrier (BBB) injury using IgG staining. Interestingly, the extent of BBB disruption was similar at week-1 for both implants but 
greater at week-8s with the stiff implants (Fig. 5, K vs L, and V). Furthermore, BBB leakage caused by the stiff implant is higher at week-8 than week-1, suggesting that the sustained mechanical disruption of the neural tissue by the tethered stiff implant results in persistent and aggravated BBB leakage. At week-1 time point, the intensity of Vimentin and L1 are higher around the stiff implant both nearby and far from the implants (Fig. 5, Q and S). At week-8, Vimentin and L1 again showed a much higher intensity near the stiff wires but the response zone is more confined to implants (Fig. 5R, T and V). Vimentin has been extensively used as a marker for inflammatory foreign body response while L1 has been shown to upregulate following implantation injury [6]. Taken together, all results indicate the superior performance of soft wires in facilitating the healing of tissue near the implants.

\section{Explanted wire and tissue integration}

Neurons ( $\beta$-tubulin III), microglia (Iba-1), and astrocytes (GFAP) were labeled to qualitatively evaluate the types of tissue attached to the soft wires and tungsten controls. Note only 1 (week-1) and 3 (week-8) soft wires were removed due to loose headcaps which separated from skull during brain dissection; the remaining soft implants were intentionally left in the brain. Therefore quantification is not possible. At both week-1 and week- 8 time points, a uniformly distributed layer of cells, including large amount of neurons, were found on soft wires (Fig. 6, E and G). In comparison, sparsely-distributed cells were located along the surface of stiff wires (Fig. 6, B, D, F, H, J, L, N and P). This result clearly indicates that soft wires greatly improve neural tissue integration of the implant compared to the control stiff wires. Scanning electron microscopy (SEM) examination of the wires following immunohistochemistry evaluation confirmed this same trend (Fig. 6, Q-T). The stripe pattern of insulation material on the stiff wires was exposed on the majority of the surfaces examined (Fig. 6, S and T) while the surface of the soft wires was covered by cells (Fig. 6, Q and R). The extent of cellular coverage was most prominent when wires were explanted at chronic time points (Fig. 6, R) in part because of the progressive condensation of the glial sheath around the implant. These results suggest that neural tissue integration is greatly improved with soft wire implants as compared to control wires.

\section{Neural stimulation with soft microwires}

To validate the in vivo electrical connectivity of the soft electrode, the electrochemical impedance spectrum was characterized in the rodent brain. Electrochemical impedance spectroscopy measures the resistance of current flow between the implanted electrode through the targeted neural tissue to the ground [24, 43]. The impedance modulus of the implanted soft wire was maintained at a functional range around $41 \mathrm{kOhm}$ at the physiologically relevant frequency of $1000 \mathrm{~Hz}$; Fig. 7A shows that the soft wire has the proper range of impedance and a smooth phase angle curve which indicates that it is a satisfactory condition. In fact, both insulation cracking and excessive fluid infiltration would lead to low electrode impedance, while 
a damaged electrical connection or a small de-insulated surface would cause high electrode impedance and noisy impedance phase angle.

To further functionally validate the soft electrode for electrical stimulation and neuromodulation, a standard DBS stimulation experiment in rat STN was conducted. The stimulation paradigm was applied through the soft wire electrode and the evoked activity was recorded by an EEG skull screw over the motor cortex [86]. The latency of the evoked neural LFP response (Fig. 7B; blue asterisk) following the electrical stimulation artifact (Fig. 7B; red asterisks) was in accordance with those previously reported [86]. DBS in the STN using the soft wire microelectrodes was able to evoke high amplitude EEG LFP signals in the ipsilateral motor cortex indicating functional viability in vivo (Fig. 7B).

\section{Discussion}

In this study, we characterized the acute and chronic tissue response to novel ultrasoft elastomeric microwire electrodes. Qualitatively, there is no adverse effect observed from the soft wire which contains several novel materials that have not been tested in the brain before. In general, the soft implants perform the same as or better than the commonly used tungsten wires. Based on these results, we conclude that the novel implant material is biocompatible when implanted in the brain tissue for at least 8 weeks.

Our hypothesis was that the softness and flexibility of the implant would significantly reduce the inflammatory tissue response and improve neuronal survival and health around the implant. To definitively prove this hypothesis, the diameter and surface chemistry of the implants were kept similar, enabling this study to specifically investigate the mechanical influence from other factors that could impact tissue response. Several studies have tried to compare the neural tissue reactions of flexible versus stiff implants, but these studies failed to control for the size of the implants or surface chemistry or both, making it difficult to draw conclusions [88, 89].

The low elastic modulus of the soft wires enabled in situ histological characterization of the intact electrode-tissue interface as the tissue could be sectioned with the microelectrode in the tissue. This property was extremely beneficial for direct characterization of cell morphology and the inflammatory tissue response immediately adjacent to the implant. The stiff implants, on the other hand, had to be removed before sectioning, and this inevitably destroyed the immediate interface. To compensate, explant analysis was done to obtain a full understanding of the electrode-tissue integration. 1 2 soft wires per group were intentionally removed during dissection and characterization of these wires provide a comprehensive comparison between soft and stiff wires. These analysis indicate the soft wire greatly enhance the electrode-tissue integration and preferentially enabled the adherence of neurons. An intimate integration would eliminate the relative movement between the implant and host tissue thereby reducing mechanical shearing and strain induced inflammation [66]. 
Inflammatory tissue response is mitigated by implant softness and flexibility

At earlier time points (i.e., week-1), both soft and stiff wires have similar levels of glial activation with only slight improvements observed with soft microelectrodes (Fig. 2, Q and S). Since the implantation of both implant types included stainless steel shuttles, the magnitude and type of initial tissue injury caused by the surgery was similar. The inflammation observed around the implants at week-1 are largely influenced by this acute stab wound injury, making it difficult to assess the mechanical effect of the different wires [41]. At week-8, when the stab wound influence is minimized, the results clearly demonstrate that our novel soft wire implants elicit reduced microglial (Fig. 2G and T) and astrocyte (Fig. 2K and V) activity. This supports the hypothesis that soft wires can greatly ameliorate the progressive development of gliosis around the implant. Since both implants were tethered on the skull, the metal implants with $10^{6}$ time higher device stiffness will generate significant micromotion relative to the brain tissue as the animal moves, while the more flexible polymer implants are likely to reduce mechanical strain experienced by the brain tissue leading to a minimized inflammatory response. Moreover, neuronal survival and health are significantly higher at the vicinity of the soft implants than the stiff implants at week-8 (Fig. 3, N). This indicates that our novel soft wires not only reduce the inflammatory response but positively impact neurons as well. Another mechanical difference between the two implants is the bulk material softness. It was known that different cell types respond differently to different mechanical moduli. Glial cells and fibroblasts prefer to grow on high stiff substrate while neurons prefer soft substrates [90-93]. Therefore, the material stiffness may be another factor that results in the differential cellular response around the implants.

\section{$B B B$ injury and tissue repair}

At week-1, the intensity increase of vimentin and L1 around the stiff implant extended far beyond the electrode-tissue interface (Fig. 5Q and S), indicating a wide range of cellular responses, while the reactive zone of soft implants is much reduced. On the other hand, the subchronic BBB injury indicated by IgG reactivity was more confined to the interface of both implant types. Therefore, we suspect that mechanisms other than inflammatory molecules leaking out of the BBB contributed to the wide activation of cellular responses. The lateral pressure from insertion of the shuttle and the micromotion of stiff wires may be transmitted across a broader range and result in a larger area of cellular responses[42, 94]. At week-8, the increase in intensity of IgG reactivity is more confined for the soft implants, which correlates with the Vimentin and L1 staining, suggesting recovery of the BBB and successful healing. On the other hand, the stiff implants show increased IgG intensity, Vimentin $100 \mu \mathrm{m}$ away from the implants, indicating chronic BBB leakage and persistent inflammation from the stiff implants even at week-8. Possibly because stiff wire continuously exert strain to adjacent tissue through micro-motion and up regulation of pro-inflammatory cytokines[35], while the soft wire adapt in its shape to dampen or even remove the pressure caused by micro-motion.

Mechanical mismatch directly impacts the local cellular microenvironment 
We postulate that when traditional stiff devices are used, a significant amount of strain is caused by the horizontal displacement of brain against the skull-anchored implant $[88,89,95$, 96], while soft wires can dampen this effect [88]. In the representative images, the asymmetrical distribution in the cell shape distortion (Fig. 3F and $\mathrm{H}$ ) could possibly be explained by a previous study reporting rigid cylindrical implant tethered to the skull causing oval scarring[97]. The author suggested the principle direction of brain movement relative to the skull of freely moving rodents may strongly influence the tissue response and the principle direction of brain movement caused more pressure to the cell body shape towards that direction[98]. Our analyses using automated algorithms confirmed that the neurons around the stiff implant had a much higher CSSI than those around the soft wires, and the CEA of cell body deformation was well aligned with predicted strain direction. While not all neuronal nuclei are naturally spherical, the large number of cells $(\mathrm{N}=12217)$ in this meta-analysis provide evidence of dramatic mechanical strain in the tissue from the accommodation of the probe volume in the presence of the stiff wire, confirming previous observations in acute studies [34, 35]. This indicates that more compliant materials (i.e., those that more closely match the mechanical properties of the target tissue) may promote the tissue equilibration following the probe volume-induced strain during and after the insertion as postulated in our previous study[80]. Fig. 6 demonstrated that tissue integration was greatly improved on the soft wire. Better electrode tissue integration on soft wire could minimize the relative movement between the wire implant and the tissue, and reduce the mechanical distortion forces towards nearby cell bodies during brain micromotion. As a result, little distortion in cell shape was observed around the soft implant. The reduced longitudinal cell body strain caused by the lower elastic modulus may improve chronic recording performance of neural implants due to the healthier cellular environment for neurons. This hypothesis will be directly evaluated in future chronic electrophysiological studies. The completeness and depth of the cell body deformation analysis with our novel automated cell shape analysis technique is impossible or hardly tractable with any conventional manual or even semi-automated image analysis algorithms. The further utilization of this technique in combination with novel automated image collection techniques could unprecedentedly accelerate the discovery of hidden mechanisms in complex biological systems.

\section{Neural stimulation capability of the ultrasoft electrodes}

Neural implant tissue compatibility and functionality are not always highly correlated $[34,99,100]$. Therefore validating the functional performance of novel neural electrode designs is critical for future applications. In Fig. 7A, the in vivo impedance phase was maintained very near $0^{\circ}$, indicating that the resistivity of the electrode dominates the impedance at the electrodetissue interface, a characteristic of the conducting polymer electrodes. This and the impedance modulus profile were clear indications that the soft wire was electrically functional in vivo and did not undergo any breakage or de-insulation. In Fig. 7B, the implanted soft wire successfully evoked strong LFP in the ipsilateral motor cortex, but the LFP peak number and shape is more complex compared to previous bipolar biphasic stimulation studies [86]. This is probably 
because the stimulation not only evoked primary EEG LFP response by stimulating STN, but also evoked neural activity along the shank of insertion shuttle used as the counter electrode. Future experiments with bi-polar stimulation electrodes design will improve the spatial specificity of neural stimulation. High frequency STN neural stimulation near $130 \mathrm{~Hz}$ is used clinically for the treatment of Parkinson's Disease (PD) as well as other episodic neural disorders like epilepsy. The degradation of DBS effectiveness over years may partially result in tissue response and replacement of conventional metal lead electrode with soft wire implants could enhance the longevity of the device. Utilizing soft wire materials in other implantable medical devices like cochlear implant and cardiac pacemakers may benefit the reduction of tissue response as well.

\section{Possible future development}

Although improving chronic recording and stimulation is the ultimate goal, the focus of this manuscript is on the histological response to test the hypothesis that implant stiffness play a critical role in inflammatory brain tissue response. The reduced foreign body response towards these soft implants demonstrates great promise for chronic neural interface applications. Despite the promising results obtained in this study, these novel soft microelectrodes require further improvement and testing before being used broadly in chronic neural recording and stimulation applications. First, longer term functional studies evaluating stability of the device composed of the novel materials in vivo will be necessary. Secondly, the current dimensions of the wire are small enough for DBS, but further miniaturization will be necessary for microstimulation and single unit recording. Furthermore, methods need to be developed to fabricate multi-channel devices. In addition, conducting polymer and nanomaterial modifications of the electrode sites to improve neural recording specificity and long-term quality can greatly expand the versatility of this device [25, 49, 101]. Additional modifications to include bioactive coatings that promote neuronal health and reduce acute inflammation may further improve electrode tissue integration $[51,53,102-105]$.

\section{Conclusions}

The chronic brain tissue response to ultrasoft conducting elastomer-based microwire neural electrodes was examined and compared to conventional stiff implants with the same surface chemistry and nearly identical dimensions. The soft wire was successfully inserted into the brain with the facilitation of a stiff shuttle and water dissolvable PEG glue. Our result indicates the following: 1) neurons at the interface of the soft electrodes showed much less mechanical strain-induced deformation and normal morphology; 2) less inflammatory gliosis was observed near the soft electrodes compared to the stiff ones; 3) significantly less chronic BBB injury and associated inflammatory response was caused by soft implants; 4) soft wires better integrated with host tissue compared to stiff wires as demonstrated by tightly bonded neurons and other cells on the explanted surface; and 5) the soft wires are effective in stimulating STN to evoke LFP in motor cortex for potential treatment of Parkinson's Disease. 


\section{Acknowledgements}

This work was sponsored by the Defense Advanced Research Projects Agency (DARPA) Contracts No. W31P4Q-08-C-0460 and W31P4Q-11-C-0134 and NIH R01 (5R01NS062019). The authors also wish to thank the Center for Biologic Imaging at the University of Pittsburgh for microscope assistance.

[1] A.B. Schwartz, X.T. Cui, D.J. Weber, D.W. Moran, Brain-controlled interfaces: movement restoration with neural prosthetics, Neuron 52(1) (2006) 205-20.

[2] J.R. Wolpaw, N. Birbaumer, D.J. McFarland, G. Pfurtscheller, T.M. Vaughan, Brain-computer interfaces for communication and control, Clin Neurophysiol 113(6) (2002) 767-91.

[3] A.B. Schwartz, Cortical neural prosthetics, Annu Rev Neurosci 27 (2004) 487-507.

[4] R.A. Andersen, E.J. Hwang, G.H. Mulliken, Cognitive neural prosthetics, Annu Rev Psychol 61 (2010) 169-90, C1-3.

[5] W. Wang, J.L. Collinger, A.D. Degenhart, E.C. Tyler-Kabara, A.B. Schwartz, D.W. Moran, D.J. Weber, B. Wodlinger, R.K. Vinjamuri, R.C. Ashmore, J.W. Kelly, M.L. Boninger, An electrocorticographic brain interface in an individual with tetraplegia, PLoS One 8(2) (2013) e55344.

[6] C.L. Kolarcik, K. Catt, E. Rost, I.N. Albrecht, D. Bourbeau, Z. Du, T.D. Kozai, X. Luo, D.J. Weber, X. Tracy Cui, Evaluation of poly(3,4-ethylenedioxythiophene)/carbon nanotube neural electrode coatings for stimulation in the dorsal root ganglion, J Neural Eng 12(1) (2015) 016008.

[7] M.A. Nicolelis, Actions from thoughts, Nature 409(6818) (2001) 403-7.

[8] T.D. Kozai, A.L. Vazquez, Photoelectric artefact from optogenetics and imaging on microelectrodes and bioelectronics: new challenges and opportunities, Journal of Materials Chemistry B (2015).

[9] L.R. Hochberg, M.D. Serruya, G.M. Friehs, J.A. Mukand, M. Saleh, A.H. Caplan, A. Branner, D. Chen, R.D. Penn, J.P. Donoghue, Neuronal ensemble control of prosthetic devices by a human with tetraplegia, Nature 442(7099) (2006) 164-71.

[10] B. Wodlinger, J.E. Downey, E.C. Tyler-Kabara, A.B. Schwartz, M.L. Boninger, J.L. Collinger, Tendimensional anthropomorphic arm control in a human brain-machine interface: difficulties, solutions, and limitations, J Neural Eng 12(1) (2015) 016011.

[11] J.L. Collinger, B. Wodlinger, J.E. Downey, W. Wang, E.C. Tyler-Kabara, D.J. Weber, A.J. McMorland, M. Velliste, M.L. Boninger, A.B. Schwartz, High-performance neuroprosthetic control by an individual with tetraplegia, Lancet 381(9866) (2013) 557-64.

[12] J.L. Collinger, M.A. Kryger, R. Barbara, T. Betler, K. Bowsher, E.H. Brown, S.T. Clanton, A.D. Degenhart, S.T. Foldes, R.A. Gaunt, F.E. Gyulai, E.A. Harchick, D. Harrington, J.B. Helder, T. Hemmes, M.S. Johannes, K.D. Katyal, G.S. Ling, A.J. McMorland, K. Palko, M.P. Para, J. Scheuermann, A.B. Schwartz, E.R. Skidmore, F. Solzbacher, A.V. Srikameswaran, D.P. Swanson, S. Swetz, E.C. Tyler-Kabara, M. Velliste, W. Wang, D.J. Weber, B. Wodlinger, M.L. Boninger, Collaborative approach in the development of highperformance brain-computer interfaces for a neuroprosthetic arm: translation from animal models to human control, Clin Transl Sci 7(1) (2014) 52-9.

[13] S.M. Wood, J.A. Jarratt, A.T. Barker, B.H. Brown, Surface electromyography using electrode arrays: a study of motor neuron disease, Muscle Nerve 24(2) (2001) 223-30.

[14] S.A. Chong, I. Benilova, H. Shaban, B. De Strooper, H. Devijver, D. Moechars, W. Eberle, C. Bartic, F. Van Leuven, G. Callewaert, Synaptic dysfunction in hippocampus of transgenic mouse models of Alzheimer's disease: a multi-electrode array study, Neurobiol Dis 44(3) (2011) 284-91. 
[15] A.G. Rouse, S.R. Stanslaski, P. Cong, R.M. Jensen, P. Afshar, D. Ullestad, R. Gupta, G.F. Molnar, D.W. Moran, T.J. Denison, A chronic generalized bi-directional brain-machine interface, J Neural Eng 8(3) (2011) 036018.

[16] J.A. Obeso, C.W. Olanow, M.C. Rodriguez-Oroz, P. Krack, R. Kumar, E. Lang A, Deep-brain stimulation of the subthalamic nucleus or the pars Interna of the globus pallidus in Parkinson's disease, The New England Journal of Medicine 345(13) (2001) 956-963.

[17] T. Loddenkemper, A. Pan, S. Neme, K.B. Baker, A.R. Rezai, D.S. Dinner, E.B. Montgomery, Jr., H.O. Luders, Deep brain stimulation in epilepsy, Journal of clinical neurophysiology : official publication of the American Electroencephalographic Society 18(6) (2001) 514-32.

[18] H.S. Mayberg, A.M. Lozano, V. Voon, H.E. McNeely, D. Seminowicz, C. Hamani, J.M. Schwalb, S.H. Kennedy, Deep Brain Stimulation for Treatment-Resistant Depression, Neuron 45(5) (2005) 651-660. [19] L. Mallet, M. Polosan, N. Jaafari, N. Baup, M.L. Welter, D. Fontaine, S.T. du Montcel, J. Yelnik, I. Chereau, C. Arbus, S. Raoul, B. Aouizerate, P. Damier, S. Chabardes, V. Czernecki, C. Ardouin, M.O. Krebs, E. Bardinet, P. Chaynes, P. Burbaud, P. Cornu, P. Derost, T. Bougerol, B. Bataille, V. Mattei, D. Dormont, B. Devaux, M. Verin, J.L. Houeto, P. Pollak, A.L. Benabid, Y. Agid, P. Krack, B. Millet, A. Pelissolo, S.S. Group, Subthalamic nucleus stimulation in severe obsessive-compulsive disorder, N Engl J Med 359(20) (2008) 2121-34.

[20] M.A. Nicolelis, D. Dimitrov, J.M. Carmena, R. Crist, G. Lehew, J.D. Kralik, S.P. Wise, Chronic, multisite, multielectrode recordings in macaque monkeys, Proc Natl Acad Sci U S A 100(19) (2003) 11041-6.

[21] C.A. Chestek, V. Gilja, P. Nuyujukian, J.D. Foster, J.M. Fan, M.T. Kaufman, M.M. Churchland, Z. Rivera-Alvidrez, J.P. Cunningham, S.I. Ryu, K.V. Shenoy, Long-term stability of neural prosthetic control signals from silicon cortical arrays in rhesus macaque motor cortex, J Neural Eng 8(4) (2011) 045005.

[22] K.D. Wise, D.J. Anderson, J.F. Hetke, D.R. Kipke, K. Najafi, Wireless implantable microsystems: Highdensity electronic interfaces to the nervous system, P leee 92(1) (2004) 76-97.

[23] T.D. Kozai, K. Catt, X. Li, Z.V. Gugel, V.T. Olafsson, A.L. Vazquez, X.T. Cui, Mechanical failure modes of chronically implanted planar silicon-based neural probes for laminar recording, Biomaterials 37 (2015) 25-39.

[24] T.D. Kozai, Z. Du, Z.V. Gugel, M.A. Smith, S.M. Chase, L.M. Bodily, E.M. Caparosa, R.M. Friedlander, X.T. Cui, Comprehensive chronic laminar single-unit, multi-unit, and local field potential recording performance with planar single shank electrode arrays, J Neurosci Methods 242C (2014) 15-40.

[25] T. Kozai, K. Catt, Z. Du, N. Kyounghwan, O. Srivannavit, R.U. Haque, J. Seymour, K. Wise, E. Yoon, X. Cui, Chronic in vivo evaluation of PEDOT/CNT for stable neural recordings, IEEE Trans Biomed Eng (2015).

[26] J.C. Williams, R.L. Rennaker, D.R. Kipke, Long-term neural recording characteristics of wire microelectrode arrays implanted in cerebral cortex, Brain research. Brain research protocols 4(3) (1999) 303-13.

[27] M.P. Ward, P. Rajdev, C. Ellison, P.P. Irazoqui, Toward a comparison of microelectrodes for acute and chronic recordings, Brain Research 1282 (2009) 183-200.

[28] C. Wang, E. Brunton, S. Haghgooie, K. Cassells, A. Lowery, R. Rajan, Characteristics of electrode impedance and stimulation efficacy of a chronic cortical implant using novel annulus electrodes in rat motor cortex, J Neural Eng 10(4) (2013) 046010.

[29] K.A. Sillay, P. Rutecki, K. Cicora, G. Worrell, J. Drazkowski, J.J. Shih, A.D. Sharan, M.J. Morrell, J. Williams, B. Wingeier, Long-term measurement of impedance in chronically implanted depth and subdural electrodes during responsive neurostimulation in humans, Brain Stimul 6(5) (2013) 718-26. [30] S.F. Lempka, S. Miocinovic, M.D. Johnson, J.L. Vitek, C.C. McIntyre, In vivo impedance spectroscopy of deep brain stimulation electrodes, J Neural Eng 6(4) (2009) 046001. 
[31] C.R. Butson, C.B. Maks, C.C. Mclntyre, Sources and effects of electrode impedance during deep brain stimulation, Clin Neurophysiol 117(2) (2006) 447-54.

[32] K.A. Sillay, J.C. Chen, E.B. Montgomery, Long-term measurement of therapeutic electrode impedance in deep brain stimulation, Neuromodulation 13(3) (2010) 195-200.

[33] M.D. Johnson, K.J. Otto, D.R. Kipke, Repeated voltage biasing improves unit recordings by reducing resistive tissue impedances, IEEE Trans Neural Syst Rehabil Eng 13(2) (2005) 160-5.

[34] T.D. Kozai, X. Li, L.M. Bodily, E.M. Caparosa, G.A. Zenonos, D.L. Carlisle, R.M. Friedlander, X.T. Cui, Effects of caspase-1 knockout on chronic neural recording quality and longevity: Insight into cellular and molecular mechanisms of the reactive tissue response, Biomaterials 35(36) (2014) 9620-34.

[35] T.D. Kozai, A.S. Jaquins-Gerstl, A.L. Vazquez, A.C. Michael, X.T. Cui, Brain tissue responses to neural implants impact signal sensitivity and intervention strategies, ACS chemical neuroscience 6(1) (2015) 4867.

[36] L.L. Rubin, J.M. Staddon, THE CELL BIOLOGY OF THE BLOOD-BRAIN BARRIER, Annual Review of Neuroscience 22(1) (1999) 11-28.

[37] T.D.Y. Kozai, T.C. Marzullo, F. Hooi, N.B. Langhals, A.K. Majewska, E.B. Brown, D.R. Kipke, Reduction of neurovascular damage resulting from microelectrode insertion into the cerebral cortex using in vivo two-photon mapping, J Neural Eng 7(4) (2010) 046011.

[38] J.N. Turner, W. Shain, D.H. Szarowski, M. Andersen, S. Martins, M. Isaacson, H. Craighead, Cerebral astrocyte response to micromachined silicon implants, Exp Neurol 156(1) (1999) 33-49.

[39] T. Roitbak, E. Sykova, Diffusion barriers evoked in the rat cortex by reactive astrogliosis, Glia 28(1) (1999) 40-8.

[40] T.D. Kozai, A.L. Vazquez, C.L. Weaver, S.G. Kim, X.T. Cui, In vivo two-photon microscopy reveals immediate microglial reaction to implantation of microelectrode through extension of processes, J Neural Eng 9(6) (2012) 066001.

[41] R. Biran, D.C. Martin, P.A. Tresco, Neuronal cell loss accompanies the brain tissue response to chronically implanted silicon microelectrode arrays, Exp Neurol 195(1) (2005) 115-26.

[42] T.D. Kozai, J.R. Eles, A.L. Vazquez, X.T. Cui, Two-photon imaging of chronically implanted neural electrodes: Sealing methods and new insights, J Neurosci Methods 258 (2016) 46-55.

[43] J.C. Williams, J.A. Hippensteel, J. Dilgen, W. Shain, D.R. Kipke, Complex impedance spectroscopy for monitoring tissue responses to inserted neural implants, J Neural Eng 4(4) (2007) 410-23.

[44] T. Saxena, L. Karumbaiah, E.A. Gaupp, R. Patkar, K. Patil, M. Betancur, G.B. Stanley, R.V.

Bellamkonda, The impact of chronic blood-brain barrier breach on intracortical electrode function, Biomaterials (2013).

[45] P.J. Rousche, D.S. Pellinen, D.P. Pivin, Jr., J.C. Williams, R.J. Vetter, D.R. Kipke, Flexible polyimidebased intracortical electrode arrays with bioactive capability, IEEE Trans Biomed Eng 48(3) (2001) 361-

71.

[46] T.D. Kozai, A.S. Jaquins-Gerstl, A.L. Vazquez, A.C. Michael, X.T. Cui, Dexamethasone retrodialysis attenuates microglial response to implanted probes in vivo, Biomaterials 87 (2016) 157-69.

[47] J.P. Seymour, D.R. Kipke, Neural probe design for reduced tissue encapsulation in CNS, Biomaterials 28(25) (2007) 3594-607.

[48] T.D. Kozai, N.B. Langhals, P.R. Patel, X. Deng, H. Zhang, K.L. Smith, J. Lahann, N.A. Kotov, D.R. Kipke, Ultrasmall implantable composite microelectrodes with bioactive surfaces for chronic neural interfaces, Nat Mater 11(12) (2012) 1065-73.

[49] Z.J. Du, X. Luo, C.L. Weaver, X.T. Cui, Poly(3,4-ethylenedioxythiophene)-ionic liquid coating improves neural recording and stimulation functionality of MEAs, Journal of Materials Chemistry C 3(25) (2015) 6515-6524.

[50] T. Kozai, N. Alba, H. Zhang, N. Kotov, R. Gaunt, X. Cui, Nanostructured Coatings for Improved Charge Delivery to Neurons, in: M.D. Vittorio, L. Martiradonna, J. Assad (Eds.), Nanotechnology and 
Neuroscience: Nano-electronic, Photonic and Mechanical Neuronal Interfacing, Springer New York, New York, NY, 2014, pp. 71-134.

[51] C.L. Kolarcik, D. Bourbeau, E. Azemi, E. Rost, L. Zhang, C.F. Lagenaur, D.J. Weber, X.T. Cui, In vivo effects of L1 coating on inflammation and neuronal health at the electrode-tissue interface in rat spinal cord and dorsal root ganglion, Acta Biomater 8(10) (2012) 3561-75.

[52] E. Azemi, C.F. Lagenaur, X.T. Cui, The surface immobilization of the neural adhesion molecule L1 on neural probes and its effect on neuronal density and gliosis at the probe/tissue interface, Biomaterials (2011).

[53] J.R. Eles, A.L. Vazquez, N.R. Snyder, C.F. Lagenaur, M.C. Murphy, T.D.Y. Kozai, X.T. Cui, Neuroadhesive L1 coating attenuates acute microglial attachment to neural electrodes as revealed by live two-photon microscopy, Biomaterials 113 (2017) 279-292.

[54] R. Khilwani, P.J. Gilgunn, T.D.Y. Kozai, X.C. Ong, E. Korkmaz, P.K. Gunalan, X.T. Cui, G.K. Fedder, O.B. Ozdoganlar, Ultra-miniature ultra-compliant neural probes with dissolvable delivery needles: design, fabrication and characterization, Biomedical Microdevices 18(6) (2016) 97.

[55] P.J. Gilgunn, R. Khilwani, T.D.Y. Kozai, D.J. Weber, X.T. Cui, G. Erdos, O.B. Ozdoganlar, G.K. Fedder, An ultra-compliant, scalable neural probe with molded biodissolvable delivery vehicle, Micro Electro Mechanical Systems (MEMS), 2012 IEEE 25th International Conference on, 2012, pp. 56-59.

[56] P.R. Patel, H. Zhang, M.T. Robbins, J.B. Nofar, S.P. Marshall, M.J. Kobylarek, T.D.Y. Kozai, N.A. Kotov, C.A. Chestek, Chronic In Vivo Stability Assessment of Carbon Fiber Microelectrode Arrays, In Review (2016).

[57] J. Subbaroyan, D.C. Martin, D.R. Kipke, A finite-element model of the mechanical effects of implantable microelectrodes in the cerebral cortex, J Neural Eng 2(4) (2005) 103-13.

[58] G.T. Fallenstein, V.D. Hulce, J.W. Melvin, Dynamic mechanical properties of human brain tissue, J Biomech 2(3) (1969) 217-26.

[59] K. Miller, K. Chinzei, G. Orssengo, P. Bednarz, Mechanical properties of brain tissue in-vivo: experiment and computer simulation, J Biomech 33(11) (2000) 1369-76.

[60] P.C. Georges, W.J. Miller, D.F. Meaney, E.S. Sawyer, P.A. Janmey, Matrices with compliance comparable to that of brain tissue select neuronal over glial growth in mixed cortical cultures, Biophys J 90(8) (2006) 3012-8.

[61] Y.B. Lu, K. Franze, G. Seifert, C. Steinhauser, F. Kirchhoff, H. Wolburg, J. Guck, P. Janmey, E.Q. Wei, J. Kas, A. Reichenbach, Viscoelastic properties of individual glial cells and neurons in the CNS, Proc Natl Acad Sci U S A 103(47) (2006) 17759-64.

[62] R. Biran, D.C. Martin, P.A. Tresco, The brain tissue response to implanted silicon microelectrode arrays is increased when the device is tethered to the skull, J Biomed Mater Res A 82(1) (2007) 169-78. [63] V.S. Polikov, P.A. Tresco, W.M. Reichert, Response of brain tissue to chronically implanted neural electrodes, J Neurosci Methods 148(1) (2005) 1-18.

[64] P. Moshayedi, G. Ng, J.C. Kwok, G.S. Yeo, C.E. Bryant, J.W. Fawcett, K. Franze, J. Guck, The relationship between glial cell mechanosensitivity and foreign body reactions in the central nervous system, Biomaterials 35(13) (2014) 3919-25.

[65] M.C. LaPlaca, D.K. Cullen, J.J. McLoughlin, R.S. Cargill, 2nd, High rate shear strain of threedimensional neural cell cultures: a new in vitro traumatic brain injury model, J Biomech 38(5) (2005) 1093-105.

[66] H. Lee, R.V. imp, W. Sun, M.E. Levenston, Biomechanical analysis of silicon microelectrode-induced strain in the brain, J Neural Eng 2(4) (2005) 81-9.

[67] J.T. Neary, Y. Kang, K.A. Willoughby, E.F. Ellis, Activation of extracellular signal-regulated kinase by stretch-induced injury in astrocytes involves extracellular ATP and P2 purinergic receptors, J Neurosci 23(6) (2003) 2348-56. 
[68] M.N. Woodroofe, G.S. Sarna, M. Wadhwa, G.M. Hayes, A.J. Loughlin, A. Tinker, M.L. Cuzner, Detection of interleukin-1 and interleukin- 6 in adult rat brain, following mechanical injury, by in vivo microdialysis: evidence of a role for microglia in cytokine production, J Neuroimmunol 33(3) (1991) 22736.

[69] W. He, R.V. Bellamkonda, A Molecular Perspective on Understanding and Modulating the Performance of Chronic Central Nervous System (CNS) Recording Electrodes, in: W.M. Reichert (Ed.), Indwelling Neural Implants: Strategies for Contending with the In Vivo Environment, Boca Raton (FL), 2008.

[70] J.B. Leach, A.K. Achyuta, S.K. Murthy, Bridging the Divide between Neuroprosthetic Design, Tissue Engineering and Neurobiology, Front Neuroeng 2 (2010) 18.

[71] T.D. Kozai, D.R. Kipke, Insertion shuttle with carboxyl terminated self-assembled monolayer coatings for implanting flexible polymer neural probes in the brain, J Neurosci Methods 184(2) (2009) 199-205.

[72] P.R. Patel, K. Na, H. Zhang, T.D. Kozai, N.A. Kotov, E. Yoon, C.A. Chestek, Insertion of linear 8.4 mum diameter 16 channel carbon fiber electrode arrays for single unit recordings, J Neural Eng 12(4) (2015) 046009.

[73] J.R. Capadona, K. Shanmuganathan, D.J. Tyler, S.J. Rowan, C. Weder, Stimuli-responsive polymer nanocomposites inspired by the sea cucumber dermis, Science 319(5868) (2008) 1370-4.

[74] T. Ware, D. Simon, C. Liu, T. Musa, S. Vasudevan, A. Sloan, E.W. Keefer, R.L. Rennaker, 2nd, W. Voit, Thiol-ene/acrylate substrates for softening intracortical electrodes, J Biomed Mater Res B Appl Biomater 102(1) (2014) 1-11.

[75] D. Khodagholy, T. Doublet, M. Gurfinkel, P. Quilichini, E. Ismailova, P. Leleux, T. Herve, S. Sanaur, C. Bernard, G.G. Malliaras, Highly conformable conducting polymer electrodes for in vivo recordings, Adv Mater 23(36) (2011) H268-72.

[76] P. Leleux, J.M. Badier, J. Rivnay, C. Benar, T. Herve, P. Chauvel, G.G. Malliaras, Conducting polymer electrodes for electroencephalography, Advanced healthcare materials 3(4) (2014) 490-3.

[77] T. Ware, D. Simon, K. Hearon, T.H. Kang, D.J. Maitland, W. Voit, Thiol-click chemistries for responsive neural interfaces, Macromol Biosci 13(12) (2013) 1640-7.

[78] J.P. Harris, J.R. Capadona, R.H. Miller, B.C. Healy, K. Shanmuganathan, S.J. Rowan, C. Weder, D.J. Tyler, Mechanically adaptive intracortical implants improve the proximity of neuronal cell bodies, J Neural Eng 8(6) (2011) 066011.

[79] J.P. Harris, A.E. Hess, S.J. Rowan, C. Weder, C.A. Zorman, D.J. Tyler, J.R. Capadona, In vivo deployment of mechanically adaptive nanocomposites for intracortical microelectrodes, J Neural Eng 8(4) (2011) 046010.

[80] C.L. Kolarcik, S.D. Luebben, S.A. Sapp, J. Hanner, N. Snyder, T.D.Y. Kozai, E. Chang, J.A. Nabity, S.T. Nabity, C.F. Lagenaur, X.T. Cui, Elastomeric and soft conducting microwires for implantable neural interfaces, Soft Matter 11(24) (2015) 4847-4861.

[81] A. Blau, A. Murr, S. Wolff, E. Sernagor, P. Medini, G. Iurilli, C. Ziegler, F. Benfenati, Flexible, allpolymer microelectrode arrays for the capture of cardiac and neuronal signals, Biomaterials 32(7) (2011) 1778-86.

[82] D.H. Kim, J. Viventi, J.J. Amsden, J. Xiao, L. Vigeland, Y.S. Kim, J.A. Blanco, B. Panilaitis, E.S. Frechette, D. Contreras, D.L. Kaplan, F.G. Omenetto, Y. Huang, K.C. Hwang, M.R. Zakin, B. Litt, J.A. Rogers, Dissolvable films of silk fibroin for ultrathin conformal bio-integrated electronics, Nat Mater 9(6) (2010) 511-7.

[83] D.C. Rodger, A.J. Fong, W. Li, H. Ameri, A.K. Ahuja, C. Gutierrez, I. Lavrov, H. Zhong, P.R. Menon, E. Meng, J.W. Burdick, R.R. Roy, V.R. Edgerton, J.D. Weiland, M.S. Humayun, Y.-C. Tai, Flexible parylenebased multielectrode array technology for high-density neural stimulation and recording, Sensors and Actuators B: Chemical 132(2) (2008) 449-460. 
[84] S. Felix, K. Shah, D. George, V. Tolosa, A. Tooker, H. Sheth, T. Delima, S. Pannu, Removable silicon insertion stiffeners for neural probes using polyethylene glycol as a biodissolvable adhesive, Conf Proc IEEE Eng Med Biol Soc 2012 (2012) 871-4.

[85] T.D.Y. Kozai, Z. Gugel, X. Li, P.J. Gilgunn, R. Khilwani, O.B. Ozdoganlar, G.K. Fedder, D.J. Weber, X.T. Cui, Chronic tissue response to carboxymethyl cellulose based dissolvable insertion needle for ultrasmall neural probes, Biomaterials 35(34) (2014) 9255-68.

[86] C. Dejean, B. Hyland, G. Arbuthnott, Cortical effects of subthalamic stimulation correlate with behavioral recovery from dopamine antagonist induced akinesia, Cereb Cortex 19(5) (2009) 1055-63.

[87] P. Limousin, P. Pollak, A. Benazzouz, D. Hoffmann, J.F. Le Bas, E. Broussolle, J.E. Perret, A.L. Benabid, Effect of parkinsonian signs and symptoms of bilateral subthalamic nucleus stimulation, Lancet 345(8942) (1995) 91-5.

[88] J.K. Nguyen, D.J. Park, J.L. Skousen, A.E. Hess-Dunning, D.J. Tyler, S.J. Rowan, C. Weder, J.R. Capadona, Mechanically-compliant intracortical implants reduce the neuroinflammatory response, J Neural Eng 11(5) (2014) 056014.

[89] A. Sridharan, J.K. Nguyen, J.R. Capadona, J. Muthuswamy, Compliant intracortical implants reduce strains and strain rates in brain tissue in vivo, J Neural Eng 12(3) (2015) 036002.

[90] A.P. Balgude, X. Yu, A. Szymanski, R.V. Bellamkonda, Agarose gel stiffness determines rate of DRG neurite extension in 3D cultures, Biomaterials 22(10) (2001) 1077-84.

[91] L.A. Flanagan, Y.E. Ju, B. Marg, M. Osterfield, P.A. Janmey, Neurite branching on deformable substrates, Neuroreport 13(18) (2002) 2411-5.

[92] P.C. Georges, P.A. Janmey, Cell type-specific response to growth on soft materials, Journal of applied physiology 98(4) (2005) 1547-53.

[93] C.-M. Lo, H.-B. Wang, M. Dembo, Y.-I. Wang, Cell Movement Is Guided by the Rigidity of the Substrate, Biophysical Journal 79(1) (2000) 144-152.

[94] C.S. Bjornsson, S.J. Oh, Y.A. Al-Kofahi, Y.J. Lim, K.L. Smith, J.N. Turner, S. De, B. Roysam, W. Shain, S.J. Kim, Effects of insertion conditions on tissue strain and vascular damage during neuroprosthetic device insertion, J Neural Eng 3(3) (2006) 196-207.

[95] L. Karumbaiah, S.E. Norman, N.B. Rajan, S. Anand, T. Saxena, M. Betancur, R. Patkar, R.V. Bellamkonda, The upregulation of specific interleukin (IL) receptor antagonists and paradoxical enhancement of neuronal apoptosis due to electrode induced strain and brain micromotion, Biomaterials (2012).

[96] Y.T. Kim, R.W. Hitchcock, M.J. Bridge, P.A. Tresco, Chronic response of adult rat brain tissue to implants anchored to the skull, Biomaterials 25(12) (2004) 2229-37.

[97] J. Thelin, H. Jorntell, E. Psouni, M. Garwicz, J. Schouenborg, N. Danielsen, C.E. Linsmeier, Implant size and fixation mode strongly influence tissue reactions in the CNS, PLoS One 6(1) (2011) e16267. [98] P. Kohler, A. Wolff, F. Ejserholm, L. Wallman, J. Schouenborg, C.E. Linsmeier, Influence of probe flexibility and gelatin embedding on neuronal density and glial responses to brain implants, PLoS One 10(3) (2015) e0119340.

[99] J.C. Barrese, N. Rao, K. Paroo, C. Triebwasser, C. Vargas-Irwin, L. Franquemont, J.P. Donoghue, Failure mode analysis of silicon-based intracortical microelectrode arrays in non-human primates, $J$ Neural Eng 10(6) (2013) 066014.

[100] D. McCreery, S. Cogan, S. Kane, V. Pikov, Correlations between histology and neuronal activity recorded by microelectrodes implanted chronically in the cerebral cortex, J Neural Eng 13(3) (2016) 036012.

[101] N. Alba, Z. Du, K. Catt, T. Kozai, X. Cui, In Vivo Electrochemical Analysis of a PEDOT/MWCNT Neural Electrode Coating, Biosensors 5(4) (2015) 618. 
[102] E. Azemi, C.F. Lagenaur, X.T. Cui, The surface immobilization of the neural adhesion molecule L1 on neural probes and its effect on neuronal density and gliosis at the probe/tissue interface, Biomaterials 32(3) (2011) 681-92.

[103] X. Cui, V.A. Lee, Y. Raphael, J.A. Wiler, J.F. Hetke, D.J. Anderson, D.C. Martin, Surface modification of neural recording electrodes with conducting polymer/biomolecule blends, J Biomed Mater Res 56(2) (2001) 261-72.

[104] X. Cui, D.C. Martin, Electrochemical deposition and characterization of poly(3,4ethylenedioxythiophene) on neural microelectrode arrays, Sensors and Actuators B: Chemical 89(1-2) (2003) 92-102.

[105] X. Cui, J. Wiler, M. Dzaman, R.A. Altschuler, D.C. Martin, In vivo studies of polypyrrole/peptide coated neural probes, Biomaterials 24(5) (2003) 777-787. 


\section{Figure Captions}

Figure 1. Illustration of fabricated parts, implant assembly, insertion and wire electrode release. From top: 1) Original needle; 2) Fabricate half-needle shuttle; 3) Mount soft/stiff wire; 4) PEG facilitated assemble; 5) Wire detach in vivo. Inset on top right corner: SEM image of fabricated soft wire tip. Scale bar $=100 \mu \mathrm{m}$.

Figure 2. NF200, Iba-1 and GFAP expression around soft and stiff implant. NF200, Iba-1 and GFAP stains for axons, microglia and astrocytes, respectively. M-P are the overlay image including Hoechst. Groups of histology from leftmost are soft wire at week-1, stiff wire at week1 , soft wire at week-8 and stiff wire at week-8. These groups are consistent through all immunohistochemistry figures. Scale bar $=100 \mu \mathrm{m}$. Q and R are normalized intensity of NF-200 expression at week-1 (animal n=7, multiple samples from one animal, outliers removed according to $2 *$ standard deviation, $\mathrm{N}=42$ histological samples quantified for soft wire, for stiff $\mathrm{N}=42$. All subsequent are denoted similarly.) and week-8(soft $\mathrm{N}=34$, stiff $\mathrm{N}=38$ ), respectively; $\mathrm{S}$ and $\mathrm{T}$ are normalized intensity of Iba- 1 expression at week-1 (soft $\mathrm{N}=45$, stiff $\mathrm{N}=46$ ) and week8 (soft $\mathrm{N}=34$, stiff $\mathrm{N}=35$ ), respectively; $\mathrm{U}$ and $\mathrm{V}$ are normalized intensity of GFAP expression at week-1(soft $\mathrm{N}=45$, stiff $\mathrm{N}=43$ ) and week-8( calculated $0 \mu \mathrm{m}$ from the electrode-tissue interface until $300 \mu \mathrm{m}$ away, with $5 \mu \mathrm{m}$ bin size.

Figure 3. Apoptotic cell death around soft and stiff implant. Cleaved Caspase 3 (Casp3) indicates apoptotic cell death and NeuN indicate neurons. Very few co-localization of Casp3 and NeuN were observed in any group. I-L are the overlay of these stainings with Hoechst. Scale bar $=100 \mu \mathrm{m} . \mathrm{M}$ and $\mathrm{N}$ are cell density count per $\mathrm{mm}^{2}$ at week-1 (soft $\mathrm{N}=14$, stiff $\mathrm{N}=13$ ) and week-8(soft $\mathrm{N}=10$, stiff $\mathrm{N}=11$ ), respectively; cell counts are calculated by automated analysis of NeuN staining and the result is binned $30 \mu \mathrm{m}$ from the electrode-tissue interface until $210 \mu \mathrm{m}$ away, with $30 \mu \mathrm{m}$ bin size. Bins starts $30 \mu \mathrm{m}$ from the interface because cell count within $30 \mu \mathrm{m}$ from the interface is too low, yielding inaccurate results.

Figure 4. Neuronal cell deformation around soft and stiff implant. A. example image of NeuN staining for week-8 around a stiff implant. Neurons on left side of the image shows significant distortion possibly caused by the micro-motion related strain from the implant. Scale bar $=100 \mu \mathrm{m}$. B. illustration of CEA and CSSI definition exemplified by a post processed cell image. Automatically segmentation yield the red dots as the pixel boundary of the cell. Dashed green line is the long axis direction and the solid green line is the length of the long axis b, solid yellow line is the length of the short axis a. solid blue line is the connection between the center of the cell and the center of the implanted wire. The cyan arc depicts the definition of CEA as the angle between dashed green line and the solid blue line. Scale bar $=5 \mu \mathrm{m}$. C and E are CEA comparison between soft and stiff wires at week-1 and week-8, respectively. D and F are CSSI comparison between soft and stiff wires at week-1 and week-8, respectively. The dashed gray line in each image represent the mean of CEA or CSSI analysis result from control images in non 
implanted cortical tissue sections. All results are binned $30 \mu \mathrm{m}$ from the electrode-tissue interface until $210 \mu \mathrm{m}$ away, with $30 \mu \mathrm{m}$ bin size.

Figure 5. Chronic BBB leakage and potential neural regeneration around implant. Vimentin (green) clearly aggregates around the implant of all types, the stiff wires cause a broad range increase of vimentin expression far from the implant as well. L1 (red) is associated with tissue response as well as possible developing oligodendrocytes for axon repair. Very strong L1 activity is observed around stiff implants at both time points. IgG (gray) indicate BBB leakage, $\operatorname{IgG}$ intensity around stiff implant was very similar to soft wire at week 1 but became much stronger at week 8 , indicating a significant chronic leakage of BBB caused by the implant stiffness. Scale bar $=100 \mu \mathrm{m}$. Q and $\mathrm{R}$ are normalized intensity of Vimentin at week-1(soft $\mathrm{N}=9$, stiff $\mathrm{N}=8$ ) and week-8( (soft $\mathrm{N}=8$, stiff $\mathrm{N}=10$ ), respectively; $\mathrm{S}$ and $\mathrm{T}$ are normalized intensity of L1 (week-1 soft $\mathrm{N}=10$, stiff $\mathrm{N}=8$, week -8 soft $\mathrm{N}=9$, stiff $\mathrm{N}=10$ ); $\mathrm{U}$ and $\mathrm{V}$ are normalized intensity of $\operatorname{IgG}$ (week-1 soft $\mathrm{N}=10$, stiff $\mathrm{N}=9$, week- 8 soft $\mathrm{N}=10$, stiff $\mathrm{N}=11$ ). Intensities are calculated 0 $\mu \mathrm{m}$ from the electrode-tissue interface until $300 \mu \mathrm{m}$ away, with $5 \mu \mathrm{m}$ bin size. $\mathrm{Q}$ and $\mathrm{S}$ demonstrate broad range increase of Vimentin and L1 around stiff implant at week-1. R, T and V all indicate a significant portion of the chronic tissue response was caused by the mechanical property of the stiff implant.

Figure 6. Cell adhesion and surface morphology of explanted soft and stiff implants. Hochest (blue), beta-III tubulin (green) and Iba-1 (red) exhibit uniform and confluent coverage on soft wires at week- 1 and week- 8 with dominant beta-III tubulin expression. In comparison, the cells on stiff wires is scattered with Iba-1 domanance especially at week-1, indicating the stiff wires have poor integration with tissue and it preferentially attracts microglia cells. Each IHC image is the reconstruction of $11 \mathrm{z}$-stack confocal microscope images. Scale bar $=100 \mu \mathrm{m}$. Q-T illustrate the wire morphology under SEM imaging. The insulation layer on stiff wire is visible at both week-1 and week-8. While soft wires integrated tissue completely covered the implant with cells in Q and R. At week-8, the soft wire not only attracted a layer of cells, but also exhibit a bulky tissue shell consisted mostly of neural cells according to beta-III tubulin intensity in $\mathrm{G}$ and overlay image $\mathrm{O}$. SEM scale bar $=5 \mu \mathrm{m}$.

Figure 7. Impedance and DBS performance of elastomer electrode. A. Impedance spectrum of elastomer electrode for neural stimulation. A thin layer of gold is sputtered on the side of conductive elastomer core to provide better conductivity. Very low impedance is recorded on the polymer electrode to prove the neural stimulation capability. B. STN DBS with soft wire evoked strong LFP in ipsilateral motor cortex recorded by a ground screw EEG. Stars denote the electrical stimulation artifacts. 
Table 1. Antibodies used for histological characterization.

\begin{tabular}{|l|l|}
\hline Antibody & Specificity \\
\hline NF200 & Mature axons \\
\hline Iba1 & Microglia/macrophages \\
\hline GFAP & Astrocytes \\
\hline Caspase-3 & Cleaved (activated) caspase-3 \\
\hline NeuN & Neuronal nuclei \\
\hline Vimentin & Immature and reactive astrocytes, microglia, endothelial cells, fibroblasts \\
\hline L1 & Neural cell adhesion molecule \\
\hline IgG & IgG protein, especially from BBB leakage \\
\hline Beta-III Tubulin & Neuron microtubules \\
\hline
\end{tabular}




\section{End View}

Side View

()
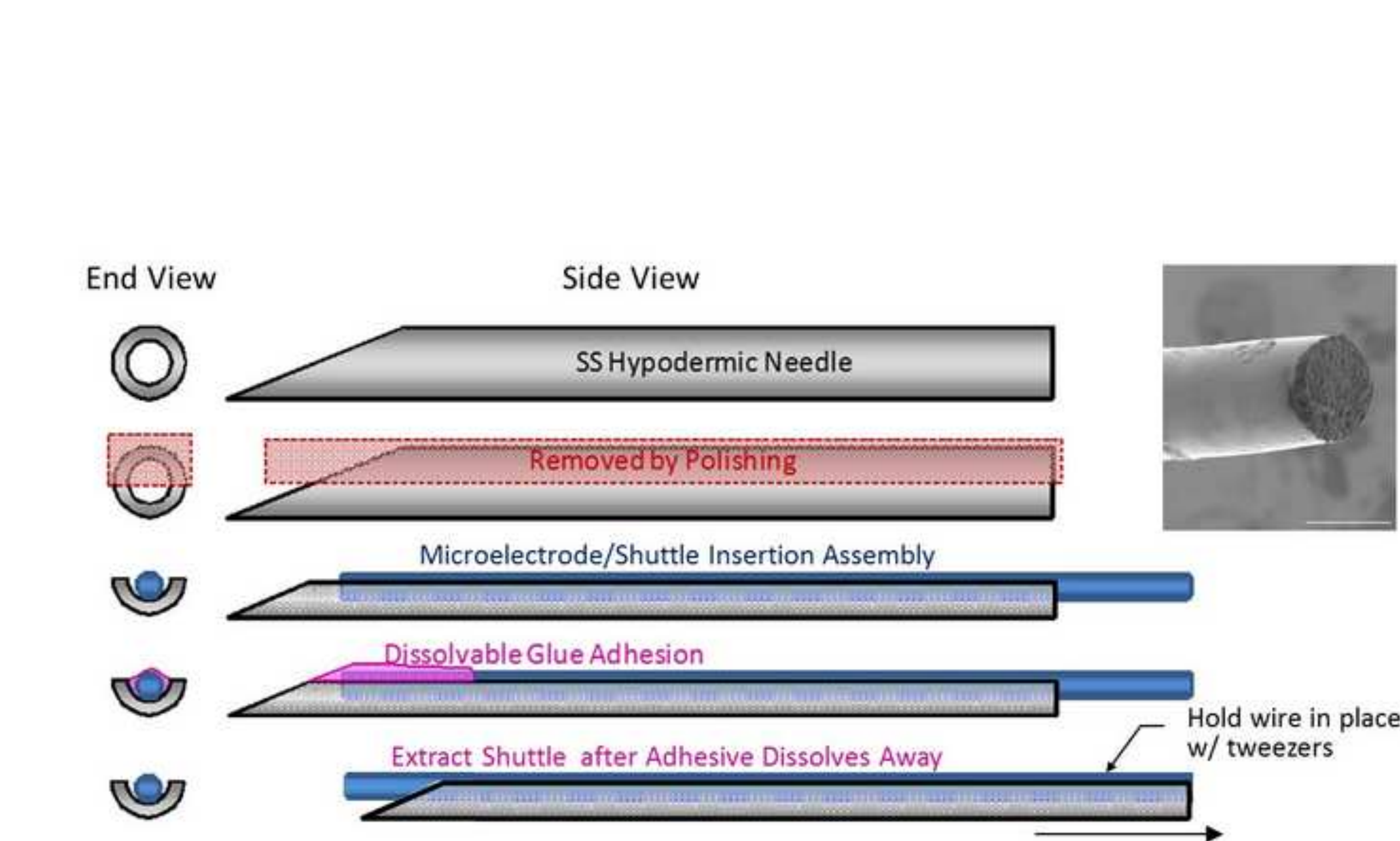

Microelectrode/Shuttle Insertion Assembly
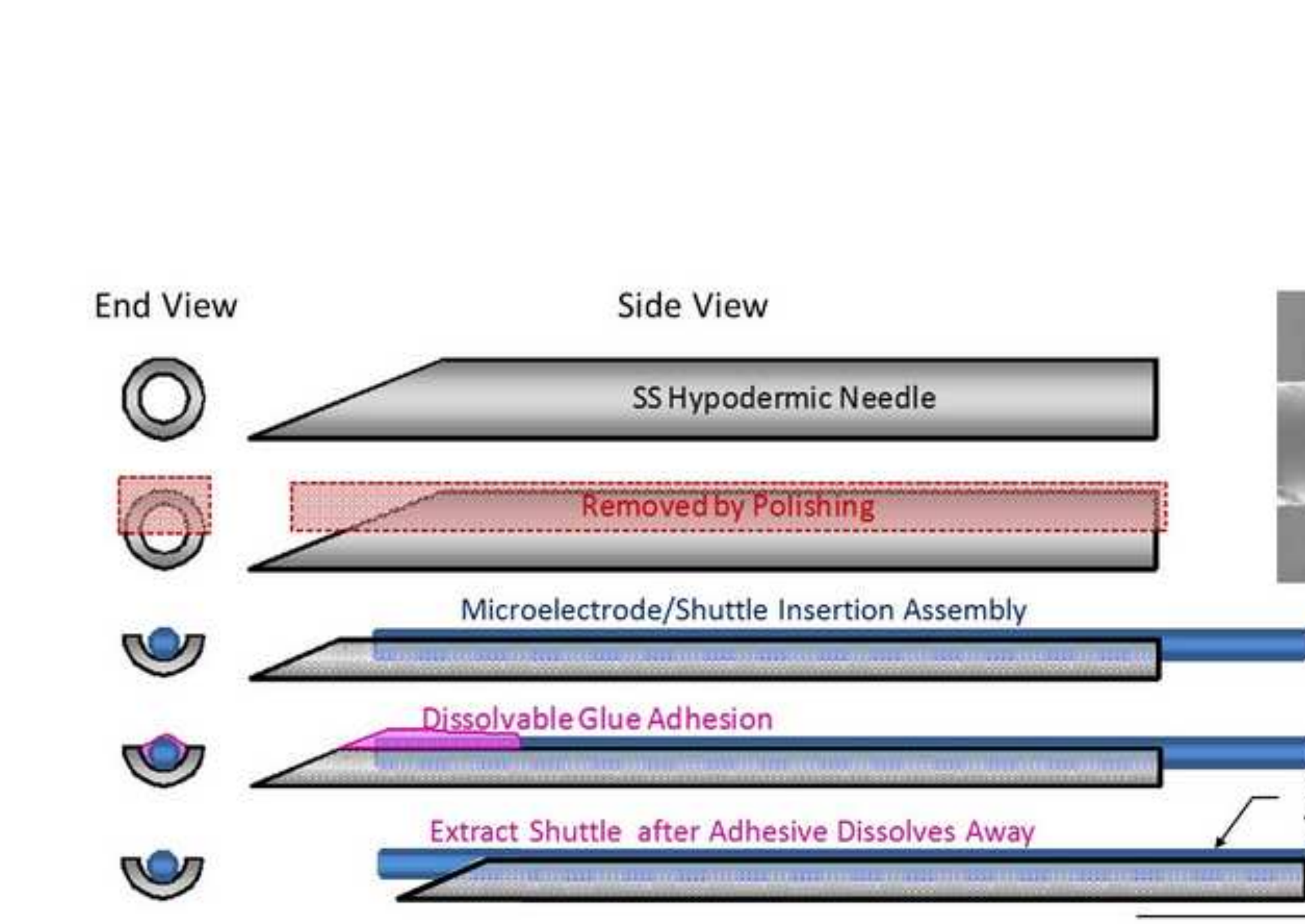

Extract Shuttle after Adhesive Dissolves Away

Hold wire in place w/ tweezers

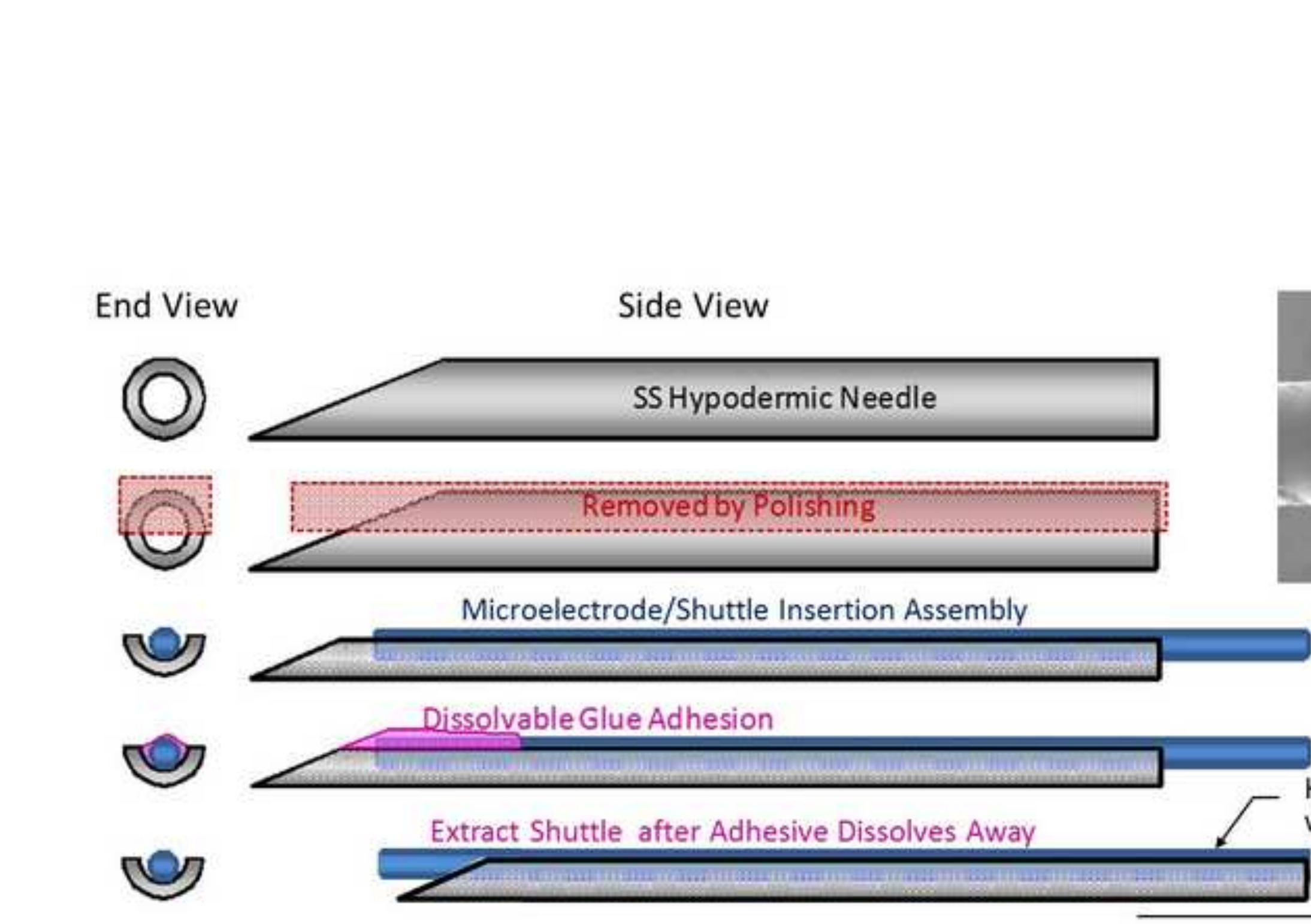




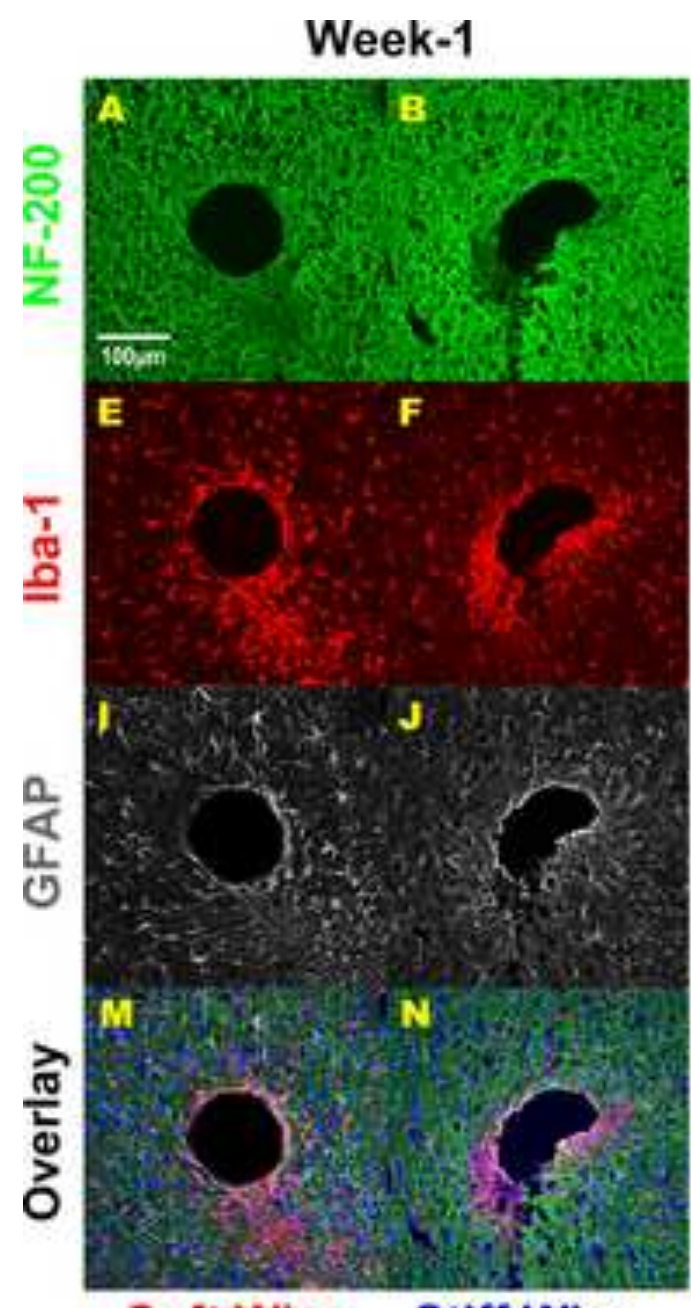

Soft Wire Stiff Wire

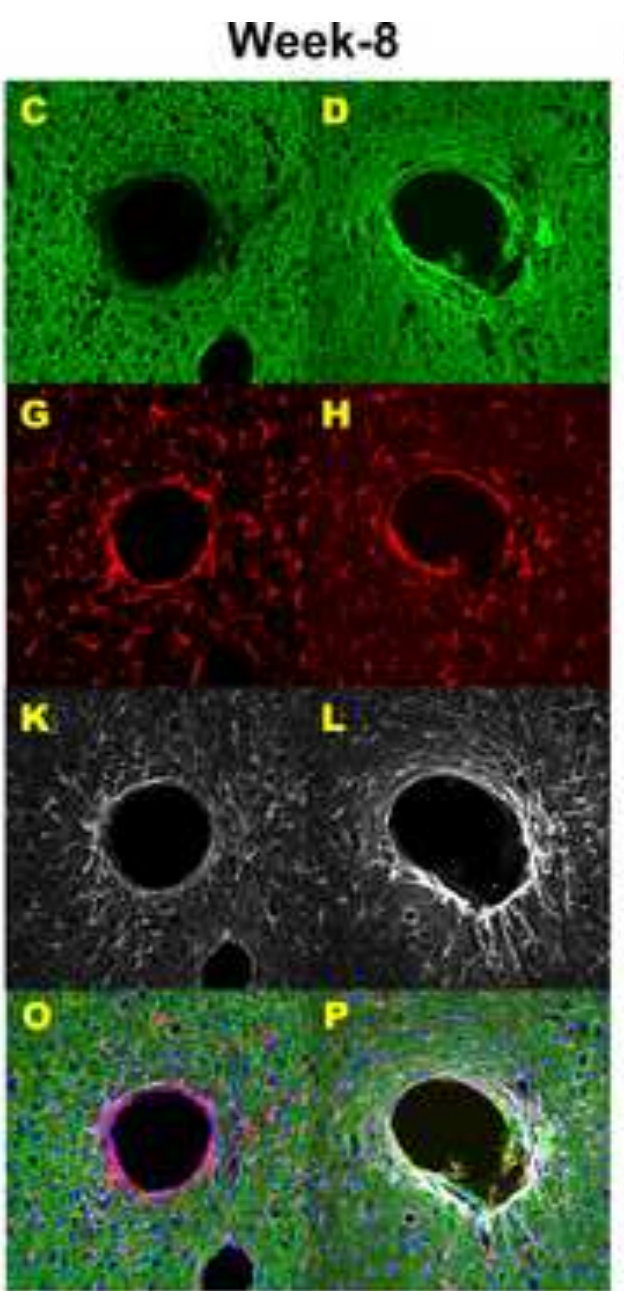

Soft Wire Stiff Wire
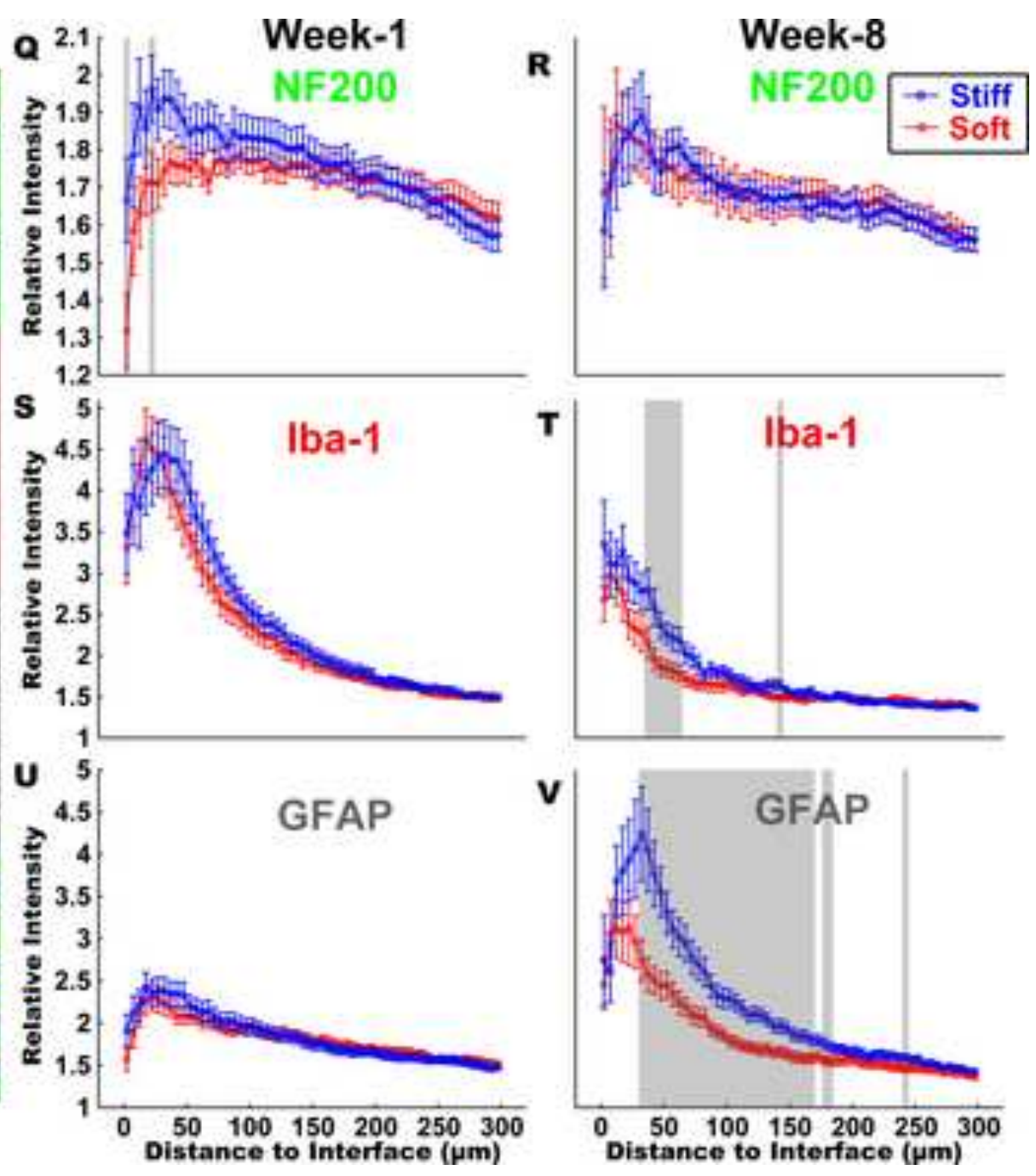
Soft Wire Stiff Wire

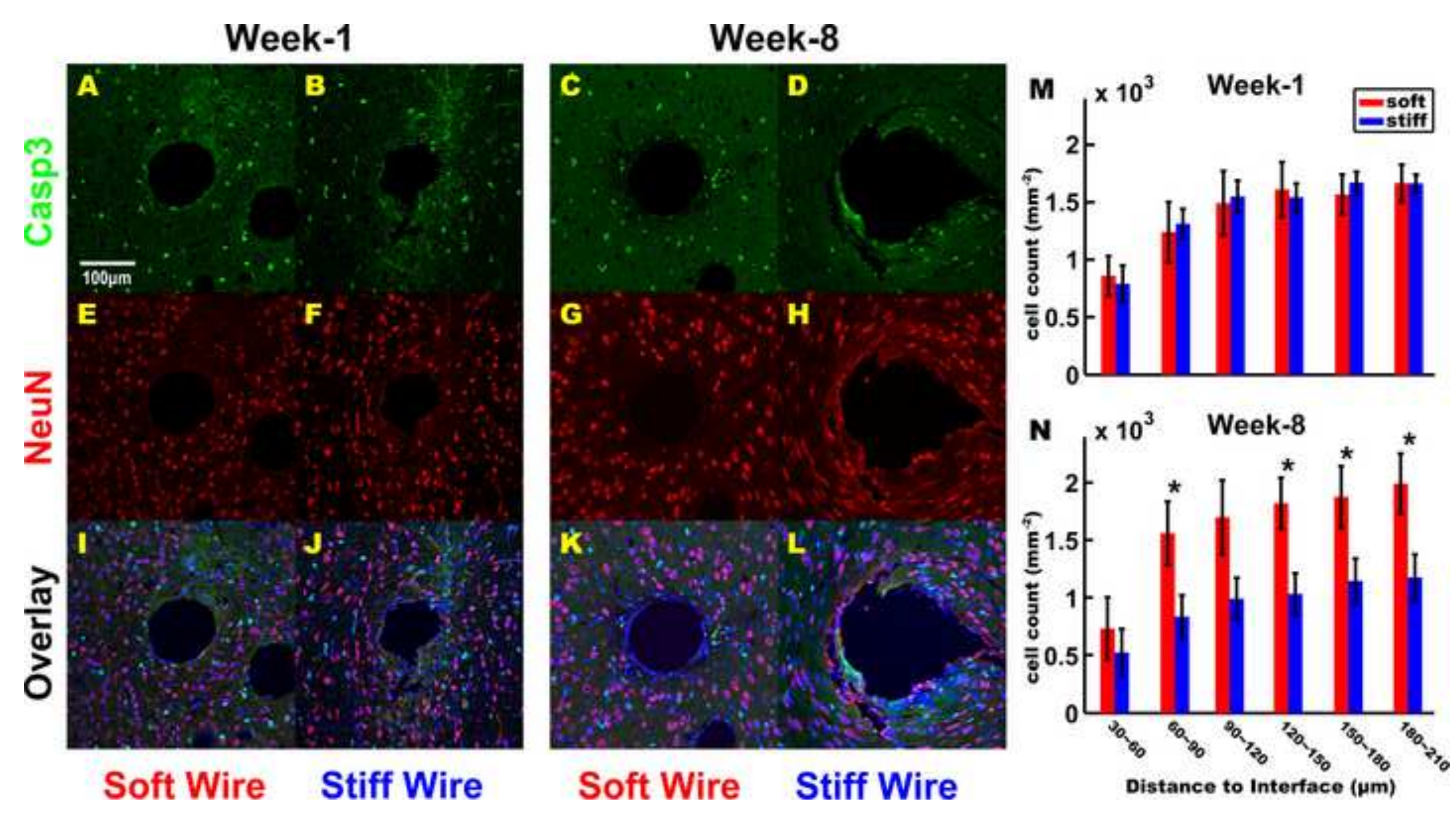

Soft Wire Stiff Wire
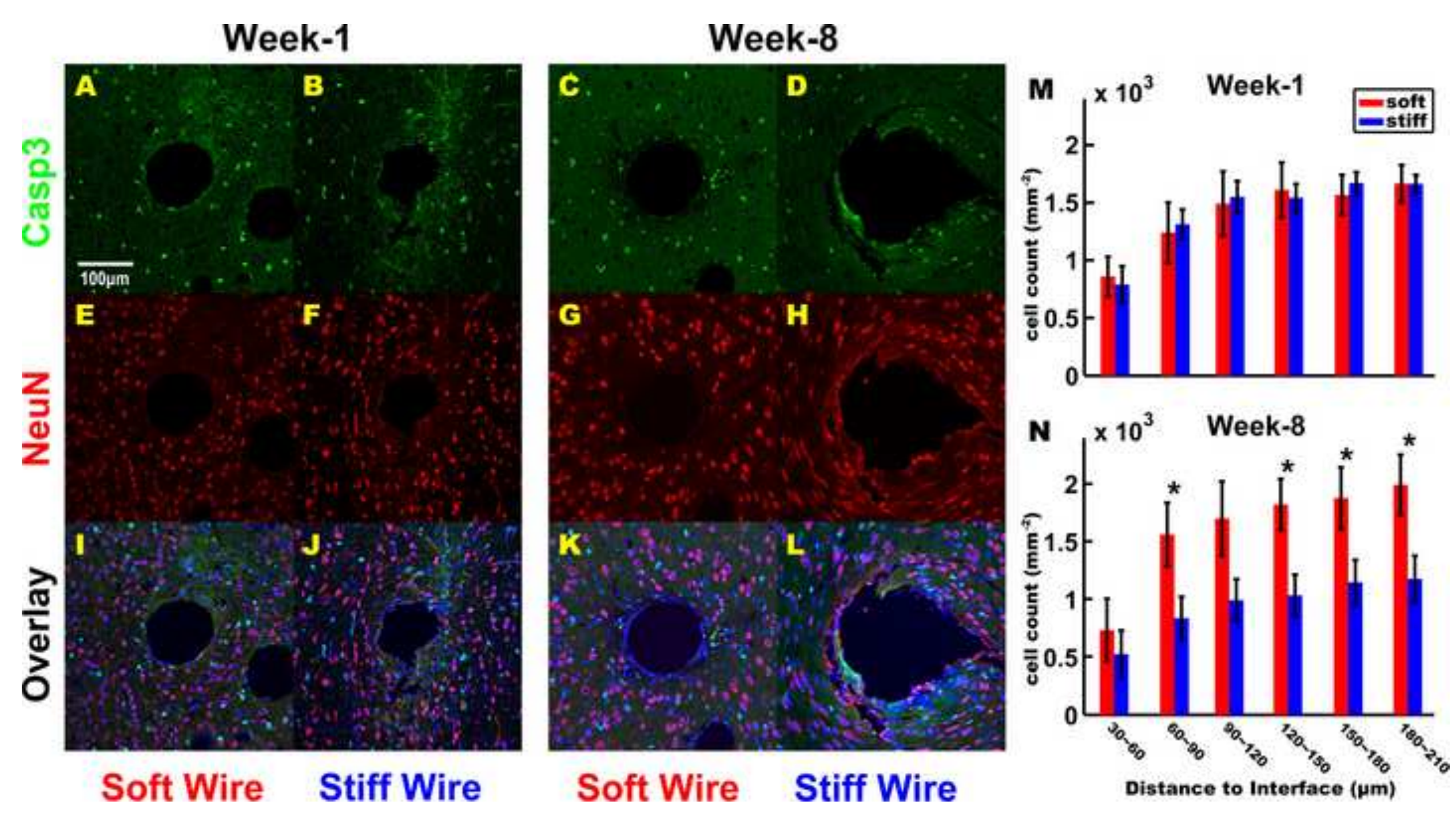

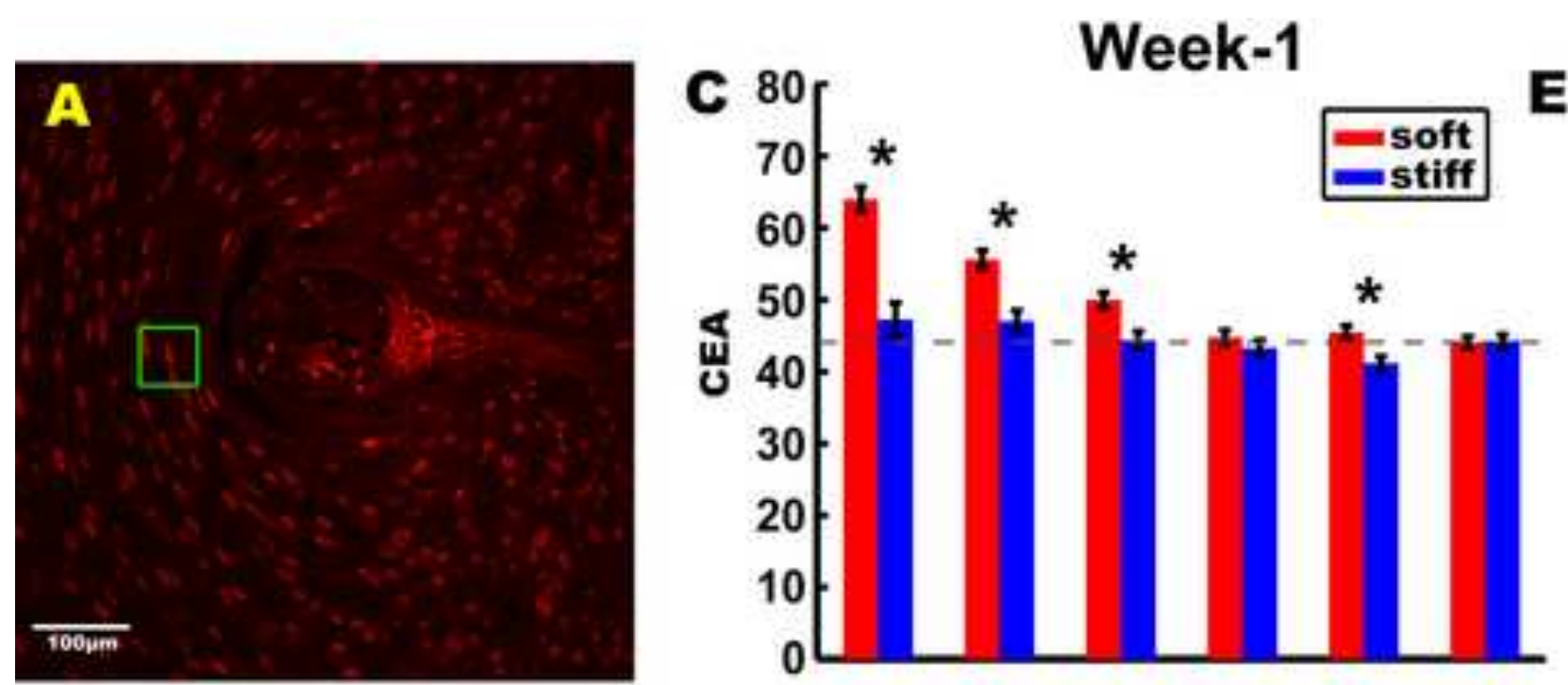

Week-8
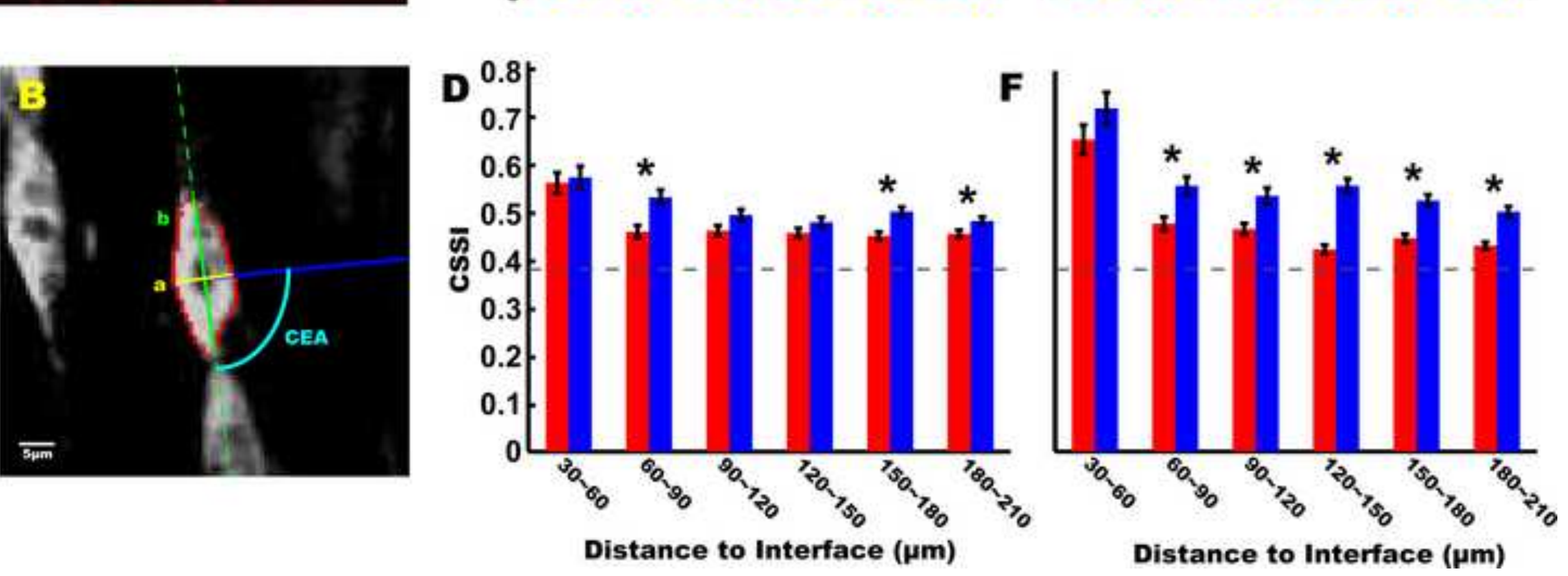


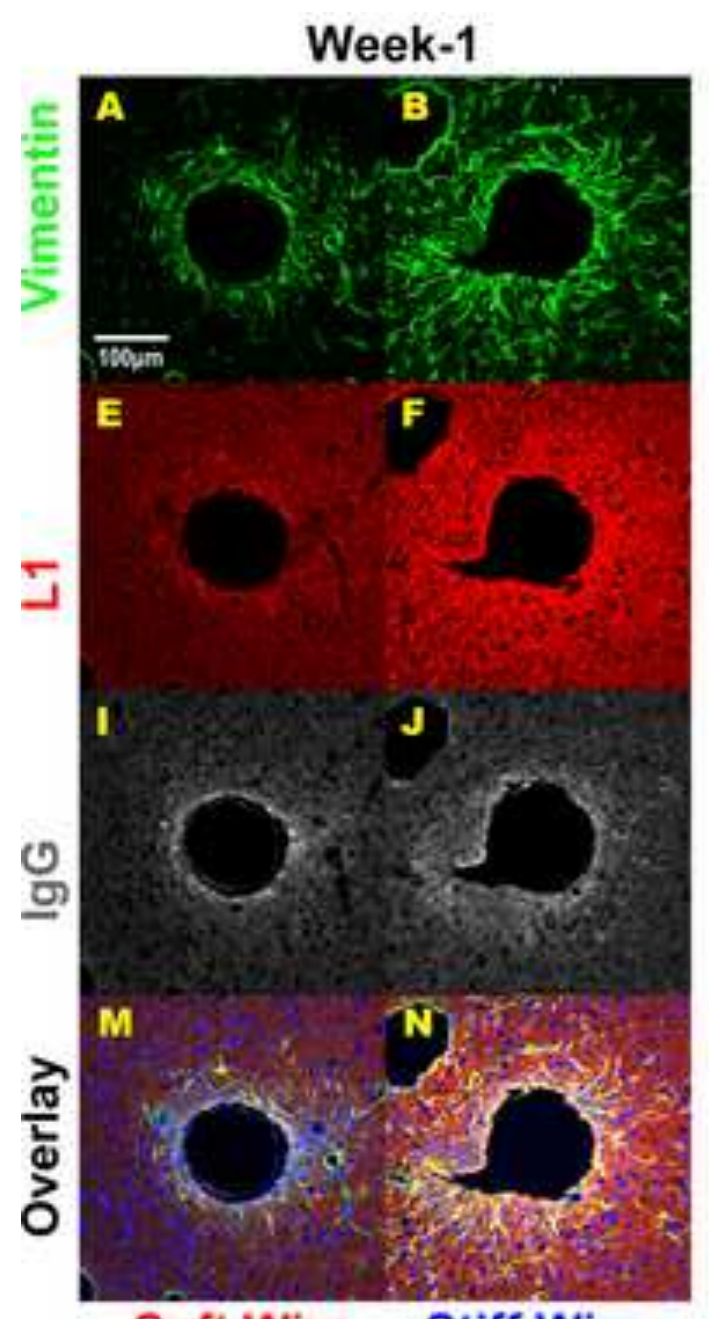

Soft Wire Stiff Wire

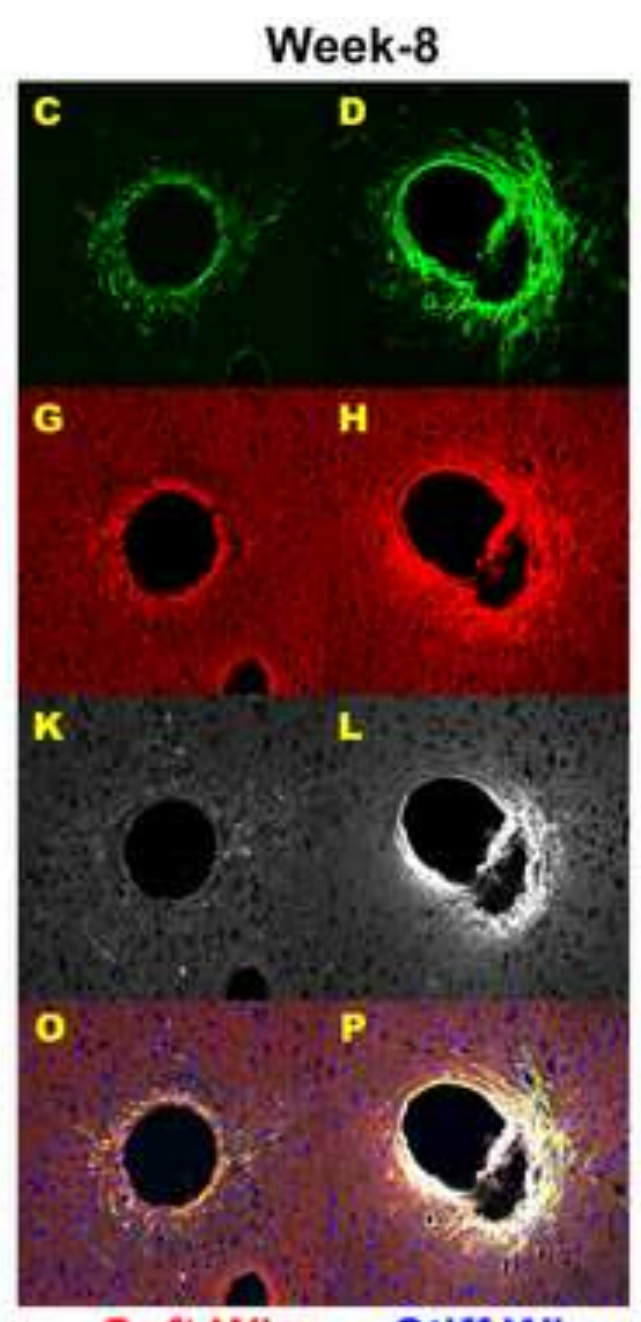

Soft Wire Stiff Wire
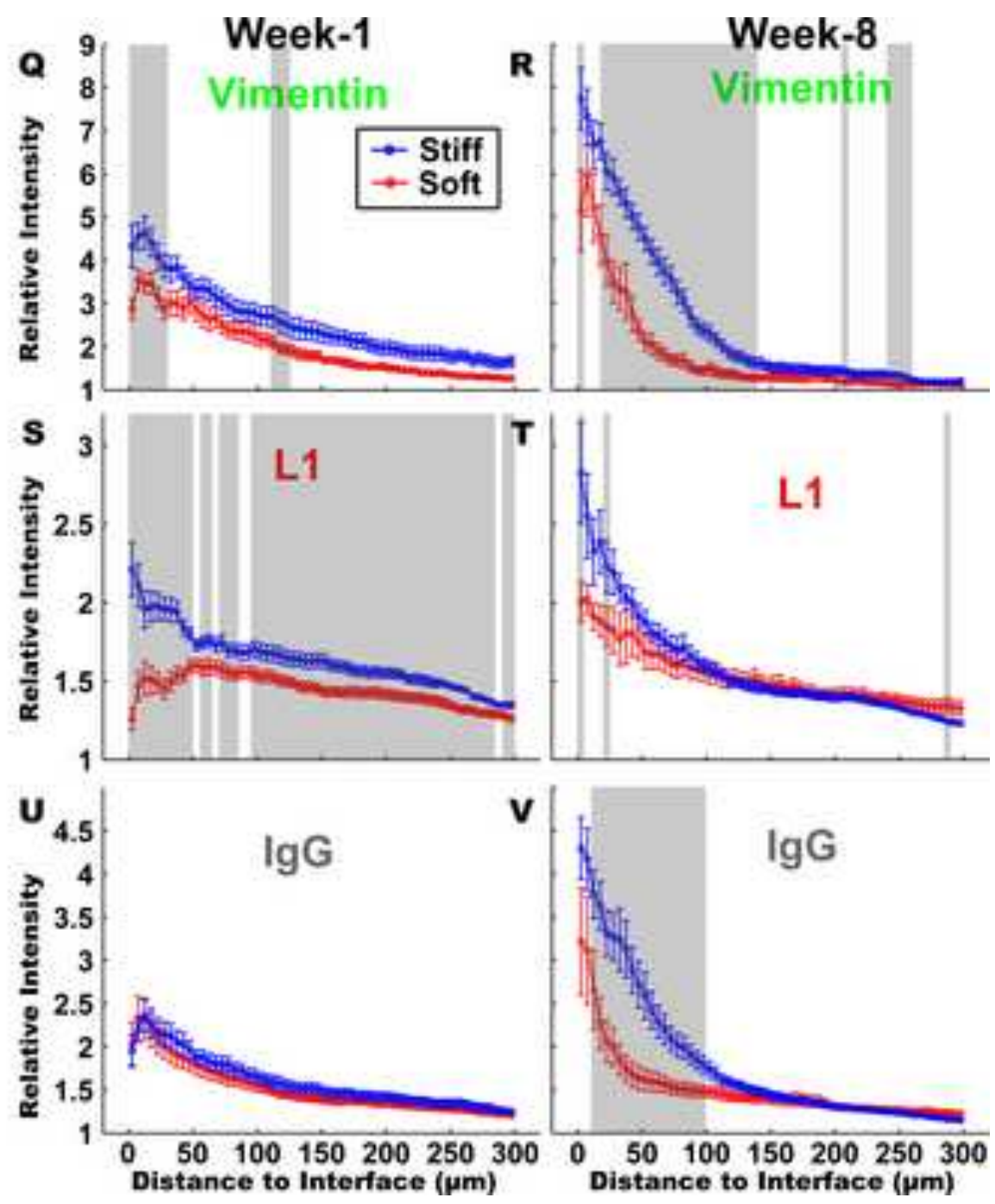

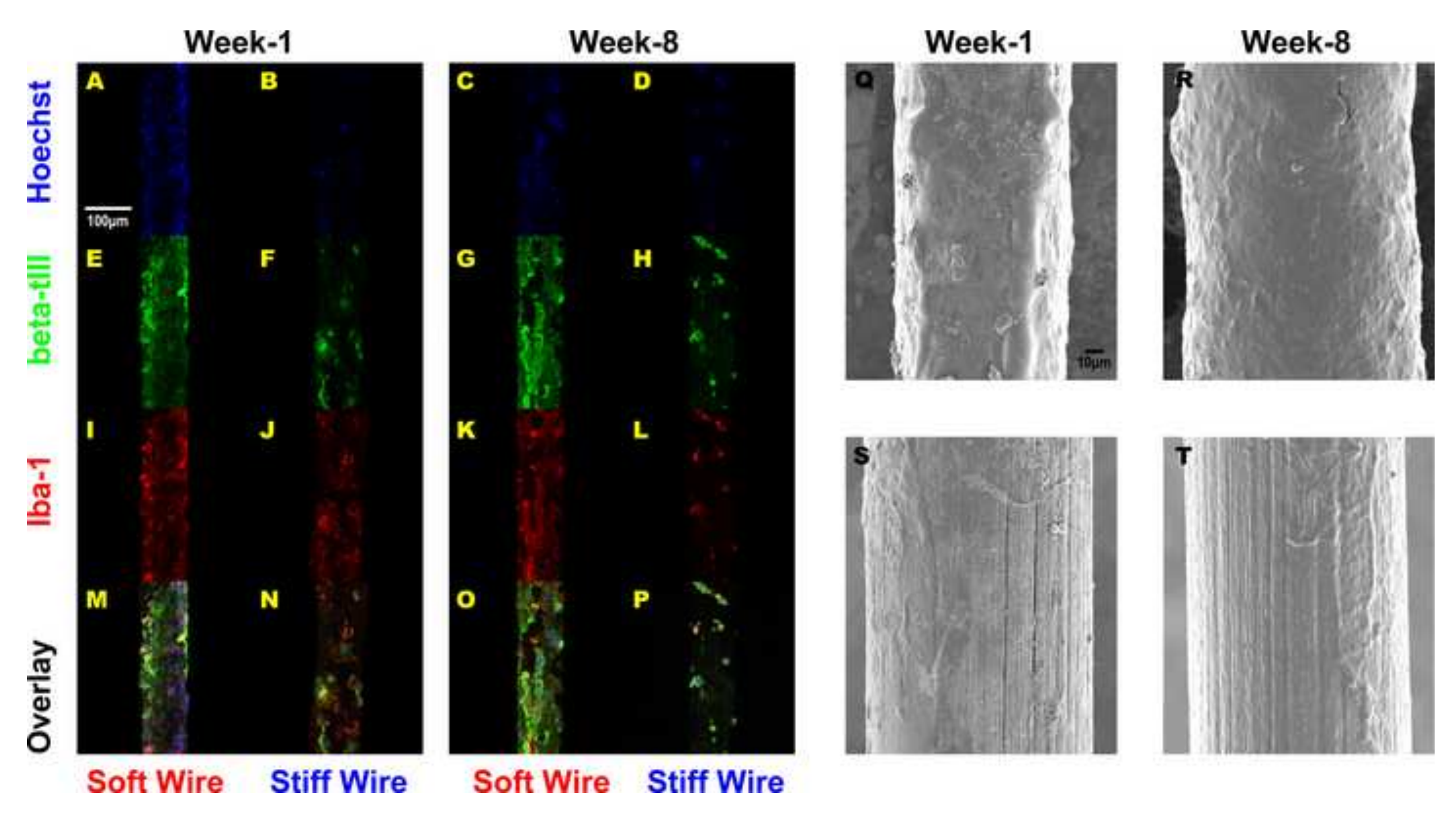

$$
\text { . }
$$



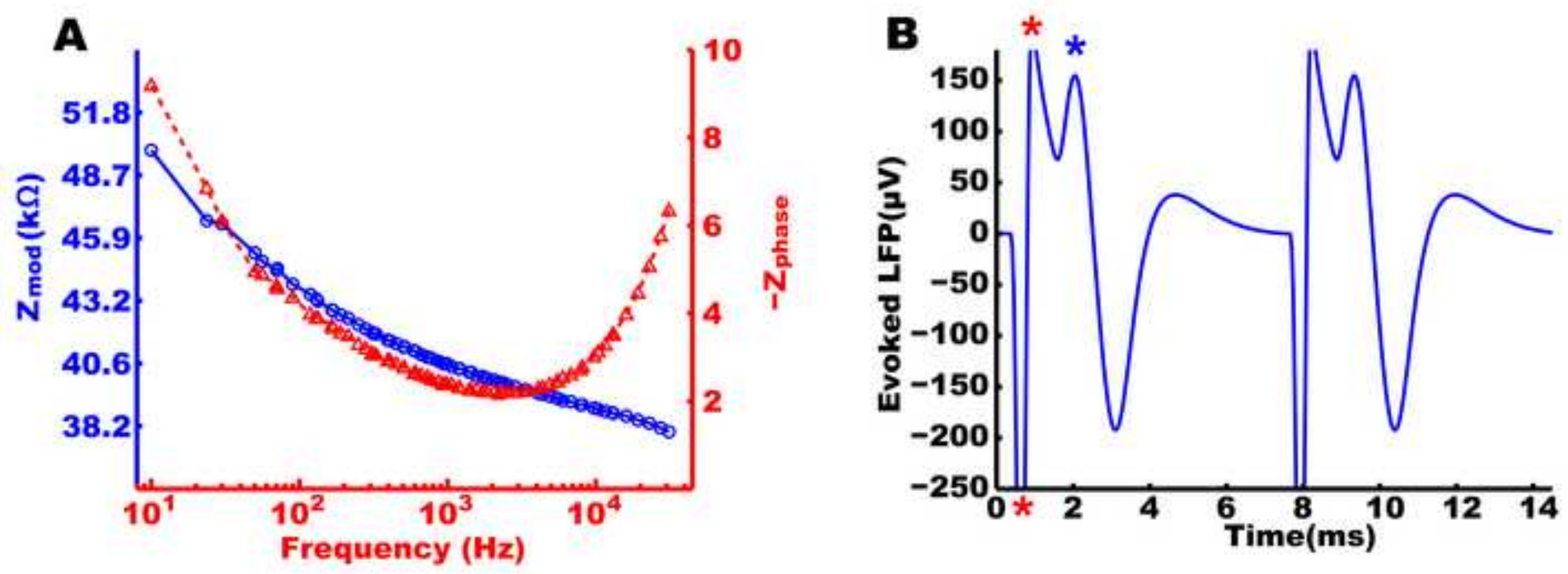


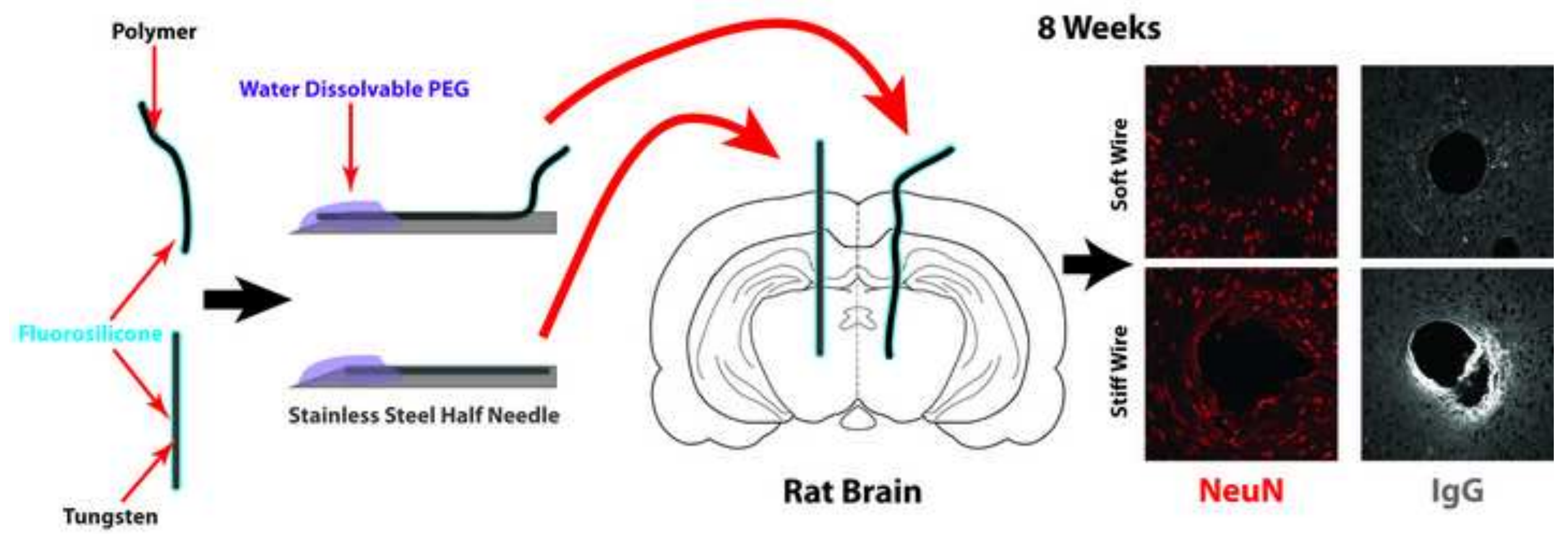


One critical challenge in translation of neural recording/stimulation electrode technology to clinically viable devices for brain computer interface (BCI) or deep brain stimulation (DBS) applications is the chronic degradation of the device performance due to inflammatory tissue reaction. While many hypothesized that soft and flexible devices elicit reduced inflammatory tissue responses, there has yet to be a rigorous comparison between soft and stiff implants. We have developed ultra-soft microelectrode with young's modulus lower than $1 \mathrm{MPa}$, closely mimicking the brain tissue modulus. Here, we present a rigorous histological comparison of this novel ultrasoft electrode and conventional stiff electrode with the same size, shape and surface chemistry, implanted in rat brain for 1-week and 8-weeks. Significant improvement was observed for ultrasoft electrodes, including inflammatory tissue reaction, electrodetissue integration as well as mechanical disturbance to nearby neurons. A full spectrum of new techniques was developed in this study, from insertion shuttle, in situ sectioning of microelectrode to automated cell shape analysis, all of which should contribute new methods to the field. Finally, we showed the electrical functionality of the ultrasoft electrode, demonstrating the potential of flexible neural implant device for future research and clinical use. 